\title{
Spectroscopic Probe Molecule Selection Using Quantum Theory, First-Principles Calculations, and Machine Learning (Supplementary Information)
}

Joshua L. Lansford ${ }^{1}$ and Dionisios G. Vlachos ${ }^{1,2 *}$

${ }^{1}$ Department of Chemical and Biomolecular Engineering, University of Delaware, 150 Academy Street, Newark, DE 19716

${ }^{2}$ Catalysis Center for Energy Innovation, University of Delaware, 221 Academy Street, Newark, DE 19716

*vlachos@udel.edu

\section{Table of Contents}

Supplementary Note 1: Breakdown of the traditional d-band model ............................... 2

Supplementary Note 2: Projection method, filling, band width, and bond energy ................ 3

Supplementary Note 3: Bond energy and band width sensitivity to unoccupied states ........ 4

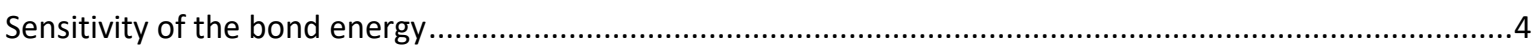

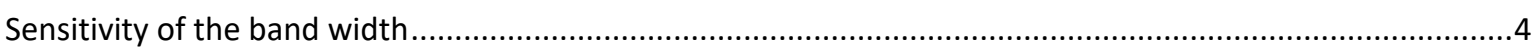

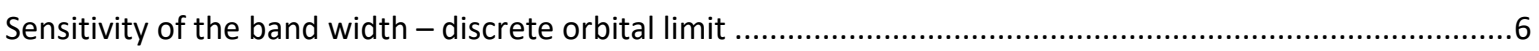

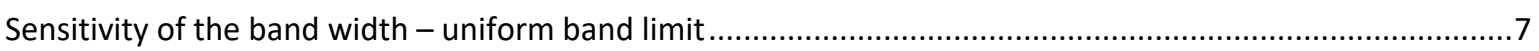

Supplementary Note 4: NO on Pt(111) at an atop site................................................... 8

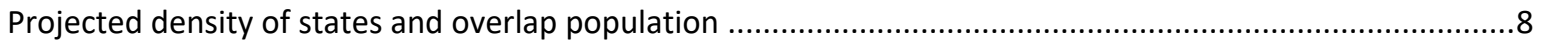

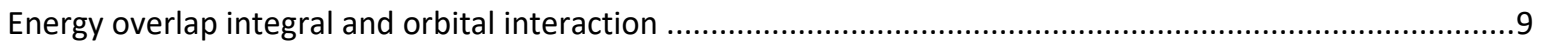

Supplementary Note 5: NO on Pt(111) at a fcc 3-fold site ............................................. 10

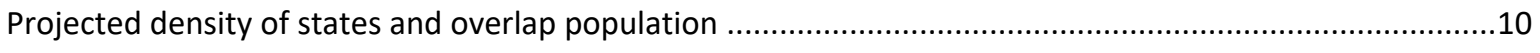

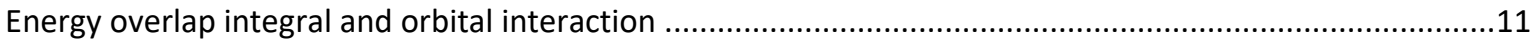

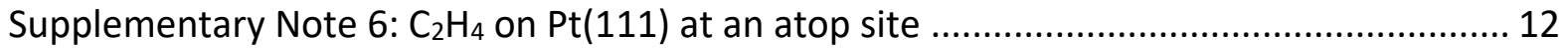

Projected density of states and molecular orbitals and overlap population ...............................................12

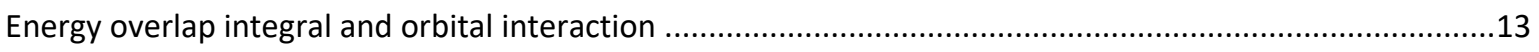

Supplementary Note 7: $\mathrm{C}_{2} \mathrm{H}_{4}$ on $\mathrm{Pt}(111)$ at a bridge site............................................... 14

Projected density of states and molecular orbitals and overlap population ...............................................14

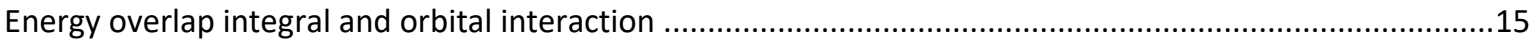

Supplementary Note 8: Gas energy shift.......................................................................... 16

Supplementary Note 9: Overview of $\mathrm{C}_{2} \mathrm{H}_{4}$ DFT data and structure model.......................... 17

Supplementary Note 10: Deriving $\chi_{\mu}(E)$ and $E_{\mu \nu}$ from COOP .................................... 18

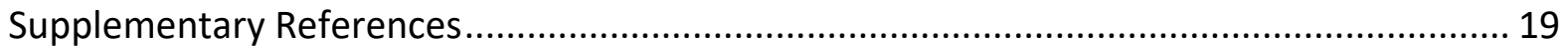




\section{Supplementary Note 1: Breakdown of the traditional d-band model}

Here we show two cases where the d-band does not correctly describe bonding or bonding changes. In Figure S1b, changes in the band center of the metal state $(\mathrm{m})$ do not necessarily cause linear changes in the adsorbate's adsorption energy.

(a)

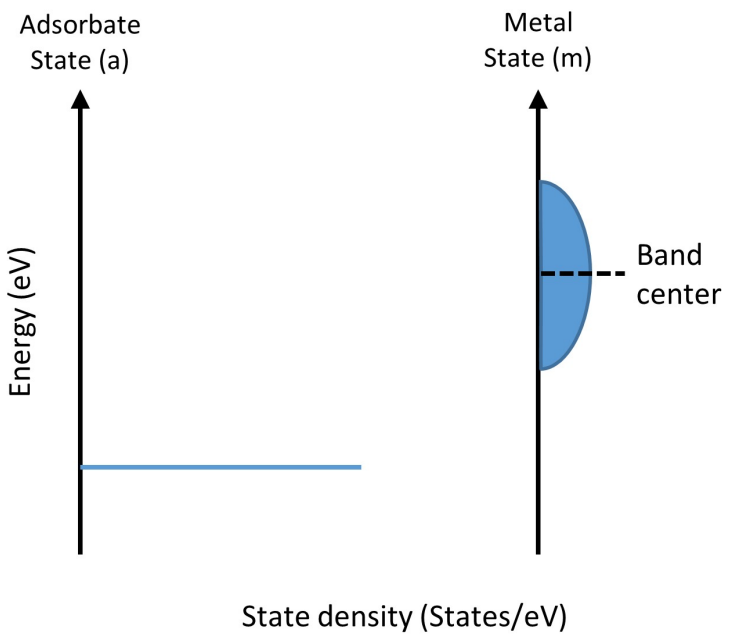

(b)
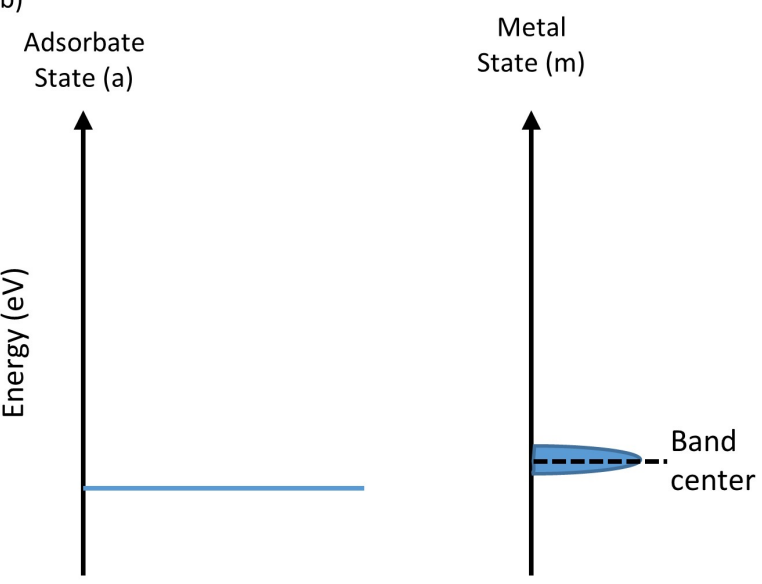

State density (States/eV)

Figure S1 Different interacting adsorbate and metal states. In (a) the metal band center is far from the adsorbate state energy and Equation 4 of the main text is valid. In (b) the mental band center is close to the adsorbate state energy and Equation 4 of the main text does not hold.

In Figure S2a and S2b, the metal state band centers are almost equivalent, and yet the bandwidths are not. In this case, the metal bandwidth describes changes in adsorbate-metal bond strengths, and changes in band-center do not.

(a)

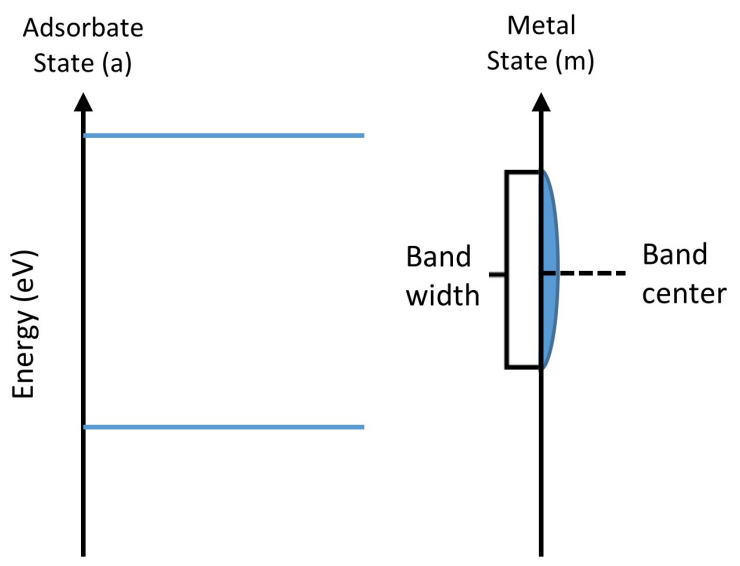

State density (States/eV) (b) Adsorbate State (a)

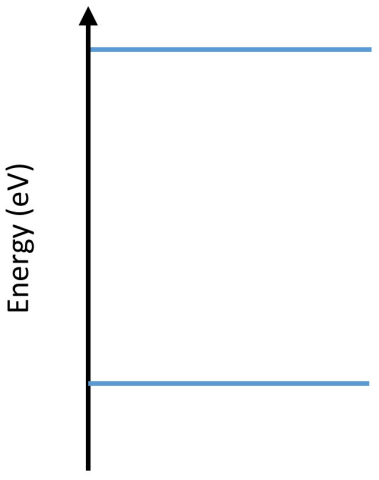

Metal State $(\mathrm{m})$

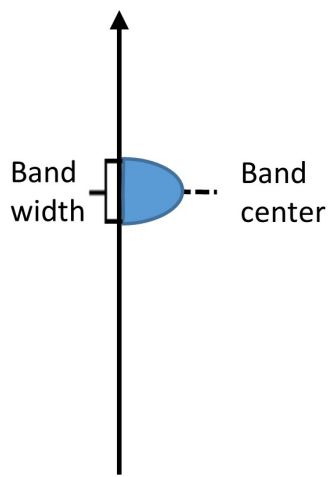

State density (States/eV)

Figure S2 Comparison of band width and band center of the metal bands to the adsorbate bonding and antibonding states. In (a) the metal band width is broad and bonding to the adsorbate states is weak. In (b) the band-width is narrow and the bonding to the adsorbate states is strong. 
Supplementary Note 2: Projection method, filling, band width, and bond energy

(a)

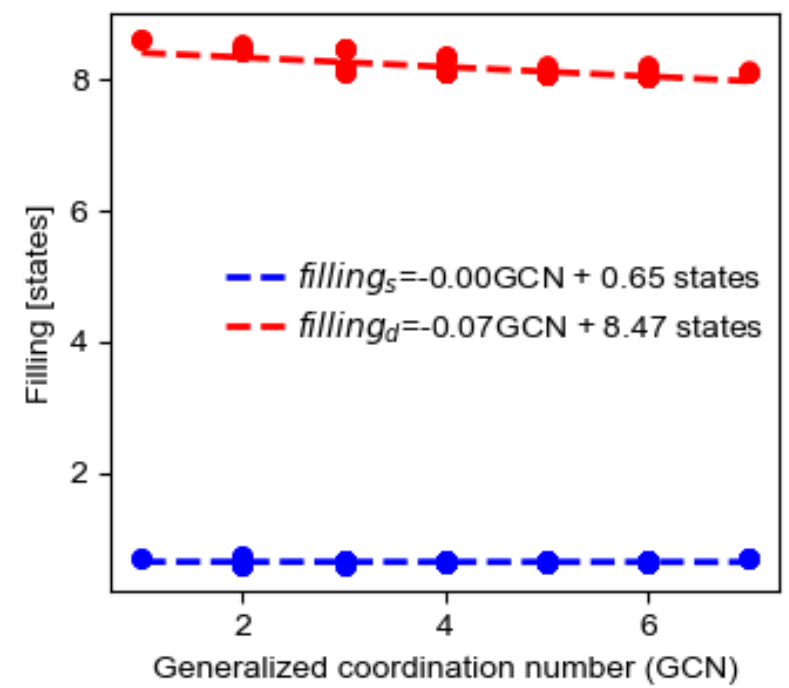

(b)

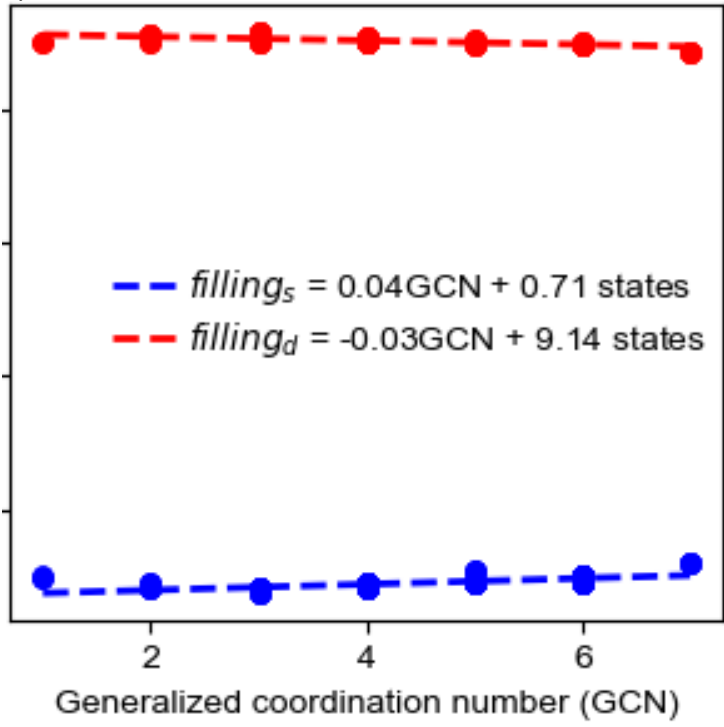

Figure S3 Orbital fillings. Shown are the integrated occupied state densities for projections from VASP (a) and LOBSTER with $7 \mathrm{~N}$ bands (b), where $\mathrm{N}$ is the number of Pt atoms, for the $\mathrm{s}$ (blue circles) and $\mathrm{d}$ (red circles) states. Also shown are the regressions of the $s$ (blue dashed lines) and $d$ (red dashed lines) fillings.

\begin{tabular}{|l|c|c|}
\hline \multicolumn{3}{|c|}{$\begin{array}{l}\text { Table S1 Integrated orbital densities for CO, determined } \\
\text { using VASP and LOBSTER. }\end{array}$} \\
\hline & \multicolumn{2}{|c|}{ Integrated States } \\
\hline State & VASP & LOBSTER \\
\hline $3 \sigma$ & 1.98 & 2.00 \\
\hline $4 \sigma$ & 1.54 & 2.00 \\
\hline $1 \pi$ & 2.76 & 4.00 \\
\hline $5 \sigma$ & 1.21 & 2.01 \\
\hline $2 \pi^{*}$ & 1.978 & 4.00 \\
\hline $6 \sigma^{*}$ & $\sim 0$ & 2.00 \\
\hline
\end{tabular}



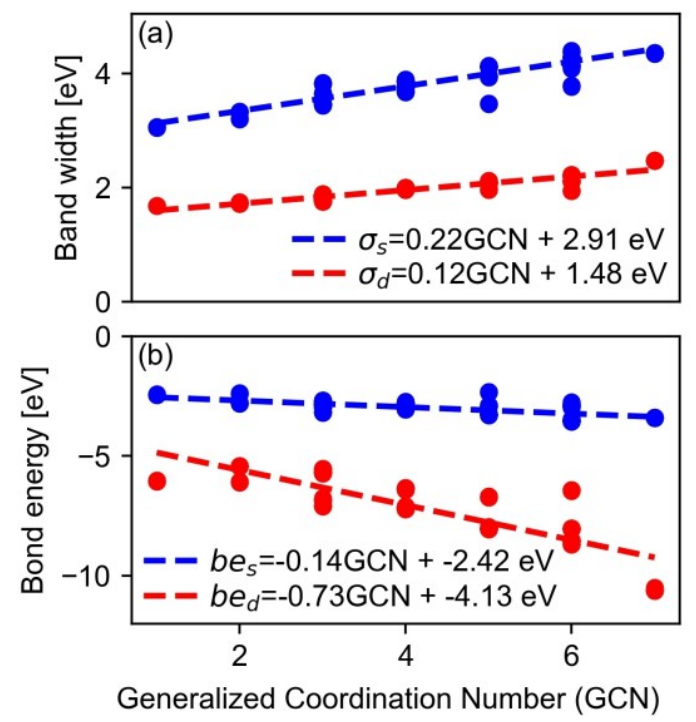

Figure S4 Band width and bond energy from VASP projections with $7 \mathrm{~N}$ bands, where $\mathrm{N}$ is the number of $\mathrm{Pt}$ atoms. Shown is the square root of the density of states' second moment (a) and tight-binding based bond energy (b) using for s (blue circles) and d (red circles) projections. Also shown are the regressions of the $s$ (blue dashed lines) and $d$ (red dashed lines) band widths and bond energies. The $\mathrm{R}^{2}$ for the $\mathrm{s}$ and $\mathrm{d}$ band widths and bond energies are 0.82 and $0.86(\mathrm{a})$, and 0.40 and $0.74(\mathrm{~b})$, respectively.

\section{Supplementary Note 3: Bond energy and band width sensitivity to unoccupied states}

Here we show that the band width, defined as the square root of the second moment of density, is less sensitive to errors in state density above the Fermi level than the tight-binding bond energy.

\section{Sensitivity of the bond energy}

Projected onto atom $i$ and band $\alpha$, the bond energy $\left(E_{\text {bond }}^{i \alpha}\right)$ given by tight-binding theory ${ }^{1-2}$ is

$$
E_{\text {bond }}^{i \alpha}=2 \int_{-\infty}^{E_{F}} \rho_{i \alpha}\left(E-\varepsilon_{i \alpha}\right) d E,
$$

where $\mathrm{E}$ is some energy, $\varepsilon_{i \alpha}$ is the band center (synonymous with orbital center), $\rho_{i \alpha}$ is the electron density projected onto orbital $\alpha$ of atom $i$, and $E_{F}$ is the fermi energy. As the upper limit of the bond energy integral is $E_{F}$, any error in the unoccupied state density above $E_{F}$ will affect the bond energy only through $\varepsilon_{i \alpha}$. Thus, the sensitivity of $E_{b o n d}^{i \alpha}$ can be captured by the total derivative of $E_{\text {bond }}^{i \alpha}$ with respect to $\varepsilon_{i \alpha}$

$$
\frac{d E_{b o n d}^{i \alpha}}{d \varepsilon_{i \alpha}}=-2 \int_{-\infty}^{E_{F}} \rho_{i \alpha} d E .
$$

Sensitivity of the band width

The band width, as defined by the second moment in $\rho_{i \alpha}\left(\mu_{i \alpha}^{(2)}\right),{ }^{2-7}$ is given by

$$
\mu_{i \alpha}^{(2)}=\int_{-\infty}^{\infty} \rho_{i \alpha}\left(E-\varepsilon_{i \alpha}\right)^{2} d E .
$$

For simplicity of the derivation we represent $\mu_{i \alpha}^{(2)}$ as a discrete integral over $\mathrm{N}$ points such that 


$$
\mu_{i \alpha}^{(2)}=\sum_{j=0}^{N} \rho_{j, i \alpha}\left(E_{j}-\varepsilon_{i \alpha}\right)^{2}
$$

As the greatest error in the projection onto atomic orbitals (often occurring as a loss in density) occurs when $j$ is close to $N$, we shall consider the sensitivity when there is some error in $\rho_{N, i \alpha}$. The total derivative of $\mu_{i \alpha}^{(2)}$ with respect to $\varepsilon_{i \alpha}$ is also dependent on $\rho_{i \alpha}$ where it changes above the $E_{F}$ such that

$$
\frac{d \mu_{i \alpha}^{(2)}}{d \varepsilon_{i \alpha}}=\frac{\partial \mu_{i \alpha}^{(2)}}{\partial \varepsilon_{i \alpha}}+\frac{\partial \mu_{i \alpha}^{(2)}}{\partial \rho_{N, i \alpha}} \frac{d \rho_{N, i \alpha}}{d \varepsilon_{i \alpha}} .
$$

The partial with respect to $\varepsilon_{i \alpha}$ is given by Equation 6

$$
\frac{\partial \mu_{i \alpha}^{(2)}}{\partial \varepsilon_{i \alpha}}=-2 \sum_{j=0}^{N} \rho_{j, i \alpha}\left(E_{j}-\varepsilon_{i \alpha}\right)
$$

and is equal to zero as

$$
\sum_{j=0}^{N} E_{j} \rho_{j, i \alpha}=\varepsilon_{i \alpha} \sum_{j=0}^{N} \rho_{j, i \alpha} .
$$

The equality in Equation 7 arises from the definition of the site and orbital projected band center

$$
\varepsilon_{i \alpha}:=\frac{\sum_{j=0}^{N} E_{j} \rho_{j, i \alpha}}{\sum_{j=0}^{N} \rho_{j, i \alpha}} .
$$

Equation 5 can be reduced to

$$
\frac{d \mu_{i \alpha}^{(2)}}{d \varepsilon_{i \alpha}}=\frac{\partial \mu_{i \alpha}^{(2)}}{\partial \rho_{N, i \alpha}} \frac{d \rho_{N, i \alpha}}{d \varepsilon_{i \alpha}} .
$$

The first part of Equation 9 is given by

$$
\frac{\partial \mu_{i \alpha}^{(2)}}{\partial \rho_{N, i \alpha}}=\left(E_{N}-\varepsilon_{i \alpha}\right)^{2} .
$$

Using the definition of the band center from Equation 8, one has

$$
\frac{d \varepsilon_{i \alpha}}{d \rho_{N, i \alpha}}=\frac{\left(\sum_{j=0}^{N} \rho_{j, i \alpha}\right) E_{N}-\left(\sum_{j=0}^{N} E_{j} \rho_{j, i \alpha}\right)}{\left(\sum_{j=0}^{N} \rho_{j, i \alpha}\right)^{2}} .
$$

Combining Equations 10 and 11, one gets

$$
\frac{d \mu_{i \alpha}^{(2)}}{d \varepsilon_{i \alpha}}=\frac{\left(E_{N}-\varepsilon_{i \alpha}\right)^{2}\left(\sum_{j=0}^{N} \rho_{j, i \alpha}\right)^{2}}{\left(\sum_{j=0}^{N} \rho_{j, i \alpha}\right) E_{N}-\left(\sum_{j=0}^{N} E_{j} \rho_{j, i \alpha}\right)} .
$$

Again using the definition of band center, we arrive at

$$
\frac{d \mu_{i \alpha}^{(2)}}{d \varepsilon_{i \alpha}}=\frac{\left(E_{N}-\varepsilon_{i \alpha}\right)^{2}\left(\sum_{j=0}^{N} \rho_{j, i \alpha}\right)^{2}}{\left(\sum_{j=0}^{N} \rho_{j, i \alpha}\right)\left(E_{N}-\varepsilon_{i \alpha}\right)},
$$

and

$$
\frac{d \mu_{i \alpha}^{(2)}}{d \varepsilon_{i \alpha}}=\left(E_{N}-\varepsilon_{i \alpha}\right)\left(\sum_{j=0}^{N} \rho_{j, i \alpha}\right) .
$$

As the binding energy is proportional to the square root of $\mu_{i \alpha}^{(2)}$, which we call the band width, we care about 


$$
\frac{d \sqrt{\mu_{i \alpha}^{(2)}}}{d \varepsilon_{i \alpha}}=\frac{1}{2}\left(E_{N}-\varepsilon_{i \alpha}\right)\left(\sum_{j=0}^{N} \rho_{j, i \alpha}\right)\left(\sum_{j=0}^{N} \rho_{j, i \alpha}\left(E_{j}-\varepsilon_{i \alpha}\right)^{2}\right)^{-\frac{1}{2}} .
$$

Substituting in Equation 2 we arrive at

$$
\frac{d \sqrt{\mu_{i \alpha}^{(2)}}}{d \varepsilon_{i \alpha}}=\frac{1}{2}\left(E_{N}-\varepsilon_{i \alpha}\right)\left(\sum_{j=0}^{N} \rho_{j, i \alpha}\right)\left(\sum_{j=0}^{N} \rho_{j, i \alpha}\left(E_{j}-\varepsilon_{i \alpha}\right)^{2}\right)^{-\frac{1}{2}} \frac{d E_{b o n d}^{i}}{d \varepsilon_{i \alpha}}\left(-2 \sum_{j=0}^{E_{F}} \rho_{j, i \alpha}\right)^{-1} .
$$

For Pt, $\alpha \in\left\{s, d_{x y}, d_{y z}, d_{z^{2}}, d_{x z}\right.$, and $\left.d_{x^{2}-y^{2}}\right\}$ and the filling $\left(\sum_{j=0}^{E_{F}} \rho_{j, i \alpha}\right)$ is close to either 1 or 1.8 for the $s$ and $\mathrm{d}$ orbitals. If we reference the energy to $\varepsilon_{i \alpha}$ and consider the entirety of the density below and above the band center to exist at $E_{0}$ and $E_{N}$, respectively, Equation 16 becomes

$$
\frac{d \sqrt{\mu_{i \alpha}^{(2)}}}{d \varepsilon_{i \alpha}}=-\frac{1}{4}\left(E_{N}\right)\left(\sum_{j=0}^{N} \rho_{j, i \alpha}\right)\left(\sum_{j=0}^{N} \rho_{j, i \alpha}\left(E_{j}\right)^{2}\right)^{-\frac{1}{2}} \frac{d E_{b o n d}^{i}}{d \varepsilon_{i \alpha}}\left(\sum_{j=0}^{E_{F}} \rho_{j, i \alpha}\right)^{-1} .
$$

There are two limiting cases; one in which $\mathrm{N}=1$ (narrow bonding and anti-bonding orbitals), and one in which $\rho_{j, i \alpha}$ is roughly constant (a band). We provide a schematic of these two limiting cases in Figure S5.

(a)

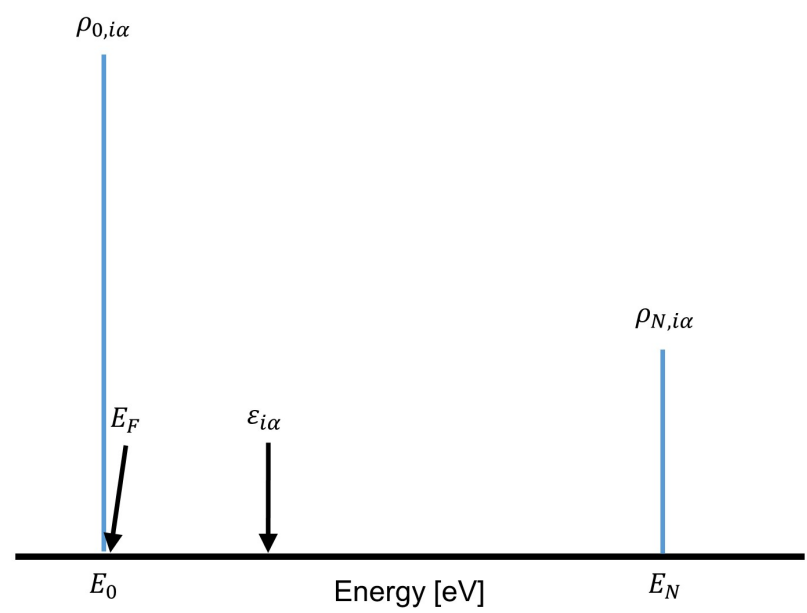

(b)

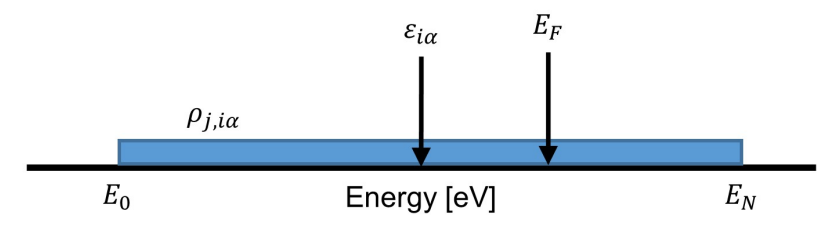

Figure S5 Schematic of narrow and broad orbitals. (a) The narrow (molecular) orbital limit in which density projected onto atom $i$ and orbital $\alpha$ is in discrete orbitals with energy $E_{0}$ and $E_{N}$ having integrated density values of $\rho_{0, i \alpha}$ and $\rho_{N, i \alpha}$. (b) The band (metallic) limit where state density projected onto atom $i$ and orbital $\alpha$ has a constant value of $\rho_{j, i \alpha}$ from $E_{0}$ to $E_{N}$ and is zero everywhere else. In both cases we reference the energy to the band center $\left(\varepsilon_{i \alpha}\right)$. The Fermi level $\left(E_{F}\right)$ is also shown.

We explore the case of discrete narrow bonding and anti-bonding orbitals for both the $s$ and $d$ states in the next subsection.

\section{Sensitivity of the band width - discrete orbital limit}

We rewrite Equation 17 as

$$
\frac{d \sqrt{\mu_{i \alpha}^{(2)}}}{d \varepsilon_{i \alpha}}=-\frac{1}{4}\left(E_{N}\right)\left(\rho_{0, i \alpha}\left(E_{0}\right)^{2}+\rho_{N, i \alpha}\left(E_{N}\right)^{2}\right)^{-\frac{1}{2}} \frac{d E_{\text {bond }}^{i}}{d \varepsilon_{i \alpha}}\left(\rho_{0, i \alpha}+\rho_{N, i \alpha}\right)\left(\rho_{0, i \alpha}\right)^{-1} .
$$

As we've set $\varepsilon_{i \alpha}$ to zero, $\rho_{0, i \alpha}\left(E_{0}\right)^{2}=\rho_{N, i \alpha}\left(E_{N}\right)^{2}=\left(E_{N}\right)^{2}$, and the sensitivity of the $s$ band width's square root is 


$$
\frac{d \sqrt{\mu_{i \alpha}^{(2)}}}{d \varepsilon_{i \alpha}}=-\frac{1}{2 \sqrt{2}} \frac{d E_{b o n d}^{i}}{d \varepsilon_{i \alpha}}
$$

For each d-orbital, we substitute $\frac{\rho_{N, i \alpha}}{\rho_{0, i \alpha}} E_{N}$ for $E_{0}$, such that

$$
\frac{d \sqrt{\mu_{i \alpha}^{(2)}}}{d \varepsilon_{i \alpha}}=-\frac{1}{4}\left(E_{N}\right)\left(\left(E_{N}\right)^{2}\left(\frac{\left(\rho_{N, i \alpha}\right)^{2}}{\rho_{0, i \alpha}}+\rho_{N, i \alpha}\right)\right)^{-\frac{1}{2}} \frac{d E_{\text {bond }}^{i}}{d \varepsilon_{i \alpha}}\left(\rho_{0, i \alpha}+\rho_{N, i \alpha}\right)\left(\rho_{0, i \alpha}\right)^{-1} .
$$

Using values of 1.8 and 0.2 for $\rho_{0, i \alpha}$ and $\rho_{N, i \alpha}$, respectively, we arrive at

$$
\frac{d \sqrt{\mu_{i \alpha}^{(2)}}}{d \varepsilon_{i \alpha}}=-0.59 \frac{d E_{\text {bond }}^{i}}{d \varepsilon_{i \alpha}}
$$

In the next section we explore the sensitivity of the band width in the uniform band limit.

Sensitivity of the band width - uniform band limit

In the case that the projected density is constant from the $E_{0}$ to $E_{N}$, the derivation benefits from converting the discrete summations to continuous integrals where there is a constant error in $\rho_{j, i \alpha}$ above the fermi level. To modify Equation 17, we must solve Equation 9 for the continuous derivative. The solution to the first part of Equation 9 becomes

$$
\frac{\partial \mu_{i \alpha}^{(2)}}{\partial \rho_{N, i \alpha}}=\frac{1}{3}\left(E_{N}^{3}-E_{F}^{3}\right)
$$

Rewriting the definition of band center as

$$
\varepsilon_{i \alpha}:=\frac{\int_{j=0}^{N} E_{j} \rho_{j, i \alpha}}{\int_{j=0}^{N} \rho_{j, i \alpha}}
$$

the total derivative of band center with respect to density above the fermi energy is

$$
\frac{d \varepsilon_{i \alpha}}{d \rho_{N, i \alpha}}=\frac{\frac{1}{2}\left(\int_{j=0}^{N} \rho_{j, i \alpha}\right)\left(E_{N}^{2}-E_{F}^{2}\right)-\left(\int_{j=0}^{N} E_{j} \rho_{j, i \alpha}\right)}{\left(\int_{j=0}^{N} \rho_{j, i \alpha}\right)^{2}},
$$

and the total derivative of the second moment due to an error in band center above the fermi energy is

$$
\frac{d \mu_{i \alpha}^{(2)}}{d \varepsilon_{i \alpha}}=\frac{\frac{1}{3}\left(E_{N}^{3}-E_{F}^{3}\right)\left(\int_{j=0}^{N} \rho_{j, i \alpha}\right)^{2}}{\frac{1}{2}\left(\int_{j=0}^{N} \rho_{j, i \alpha}\right)\left(E_{N}^{2}-E_{F}^{2}\right)} .
$$

and

$$
\frac{d \mu_{i \alpha}^{(2)}}{d \varepsilon_{i \alpha}}=\frac{2}{3} \frac{\left(E_{N}^{3}-E_{F}^{3}\right)}{\left(E_{N}^{2}-E_{F}^{2}\right)}\left(\int_{j=0}^{N} \rho_{j, i \alpha}\right) .
$$

Equation 17 for the continuous case becomes

$$
\frac{d \sqrt{\mu_{i \alpha}^{(2)}}}{d \varepsilon_{i \alpha}}=-\frac{1}{4}\left(\frac{2}{3} \frac{\left(E_{N}^{3}-E_{F}^{3}\right)}{\left(E_{N}^{2}-E_{F}^{2}\right)}\right)\left(\rho_{j, i \alpha} \int_{j=0}^{N}\left(E_{j}\right)^{2}\right)^{-\frac{1}{2}} \frac{d E_{b o n d}^{i}}{d \varepsilon_{i \alpha}}\left(\int_{j=0}^{N} \rho_{j, i \alpha}\right)\left(\int_{j=0}^{E_{F}} \rho_{j, i \alpha}\right)^{-1} .
$$

As the energy is referenced to the band center, $\rho_{j, i \alpha}=\frac{2}{2 E_{N}}, E_{0}=-E_{N}$, and 


$$
\frac{d \sqrt{\mu_{i \alpha}^{(2)}}}{d \varepsilon_{i \alpha}}=-\frac{1}{3}\left(\frac{\left(E_{N}^{3}-E_{F}^{3}\right)}{\left(E_{N}^{2}-E_{F}^{2}\right)}\right)\left(\frac{1}{3} E_{N}^{2}\right)^{-\frac{1}{2}} \frac{d E_{b o n d}^{i}}{d \varepsilon_{i \alpha}}\left(\int_{j=0}^{E_{F}} \rho_{j, i \alpha}\right)^{-1},
$$

For the s states, $E_{F}=0$, and

$$
\frac{d \sqrt{\mu_{i \alpha}^{(2)}}}{d \varepsilon_{i \alpha}}=-\frac{\sqrt{3}}{3} \frac{d E_{b o n d}^{i}}{d \varepsilon_{i \alpha}} .
$$

For the d states, $E_{F}=0.8 E_{N}$, and

$$
\frac{d \sqrt{\mu_{i \alpha}^{(2)}}}{d \varepsilon_{i \alpha}}=-\frac{\sqrt{3}}{3.98} \frac{d E_{b o n d}^{i}}{d \varepsilon_{i \alpha}} .
$$

\section{Supplementary Note 4: NO on $\mathrm{Pt}(\mathbf{1 1 1})$ at an atop site}

Projected density of states and overlap population
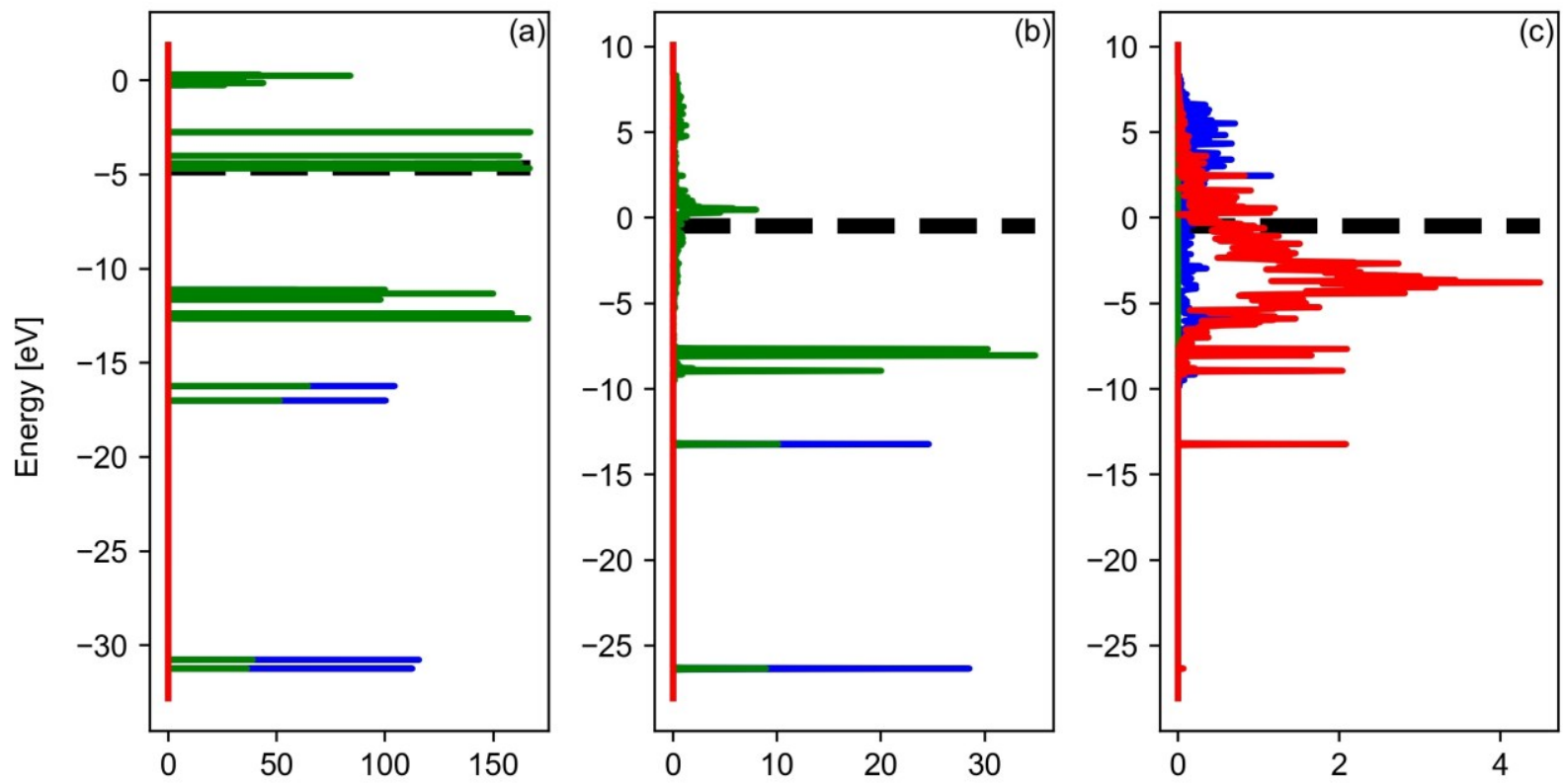

State density [states/eV]

Figure S6 Projected density of states for NO at an atop site of Pt(111). Projected density of states for gas NO (a) compared to the projected density of $\mathrm{NO}$ at an atop site (b) and that of the adsorption site after adsorption (c). Molecular orbitals of adsorbed NO in (b) have orbital energy centers located at $-26.34 \mathrm{eV},-13.23 \mathrm{eV},-8.90 \mathrm{eV},-8.04 \mathrm{eV},-7.67 \mathrm{eV}$, and $0.44 \mathrm{eV}$ with integrated state densities of 2.00, 1.85, 1.72, 1.87, 1.84, and 6.72, respectively. Molecular orbitals are labeled in (a) and (b). Density projected onto $\mathrm{s}, \mathrm{p}$, and d atomic orbitals are colored blue, green, and red, respectively. The fermi energy is given by the black dashed line. 

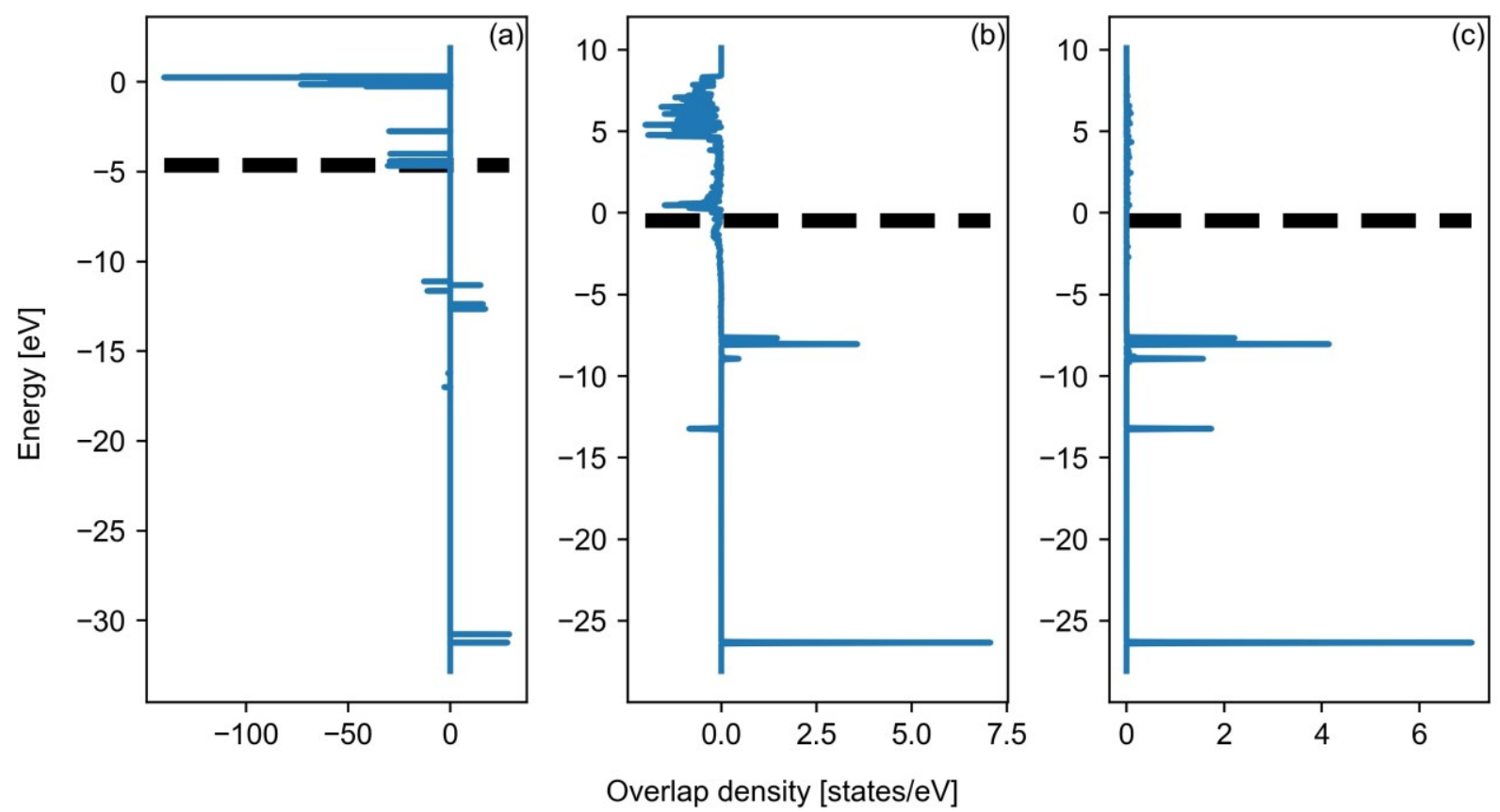

Figure S7 Crystal orbital overlap population analysis for NO on Pt(111) at an atop site. Shown are the overlap populations for gas NO (a), NO adsorbed at the atop site (b), and the sum of the positive overlaps for the adsorbed NO and its overlap with the Pt adsorption site (c). The integrated overlap populations for molecular orbitals identified in Figure $4 b(1,2,3,4,5$, and 6$)$ are 0.38 , $-0.04,0.04,0.19,0.08$, and -0.25 states up to the fermi level for adsorbed NO (b), and $0.38,0.09,0.12,0.22,0.12$, and 0.02 states for the positive overlap of the combined NO and adsorption site, respectively. The overlap is given by the blue line and the fermi energy is given by the black dashed line.

Energy overlap integral and orbital interaction
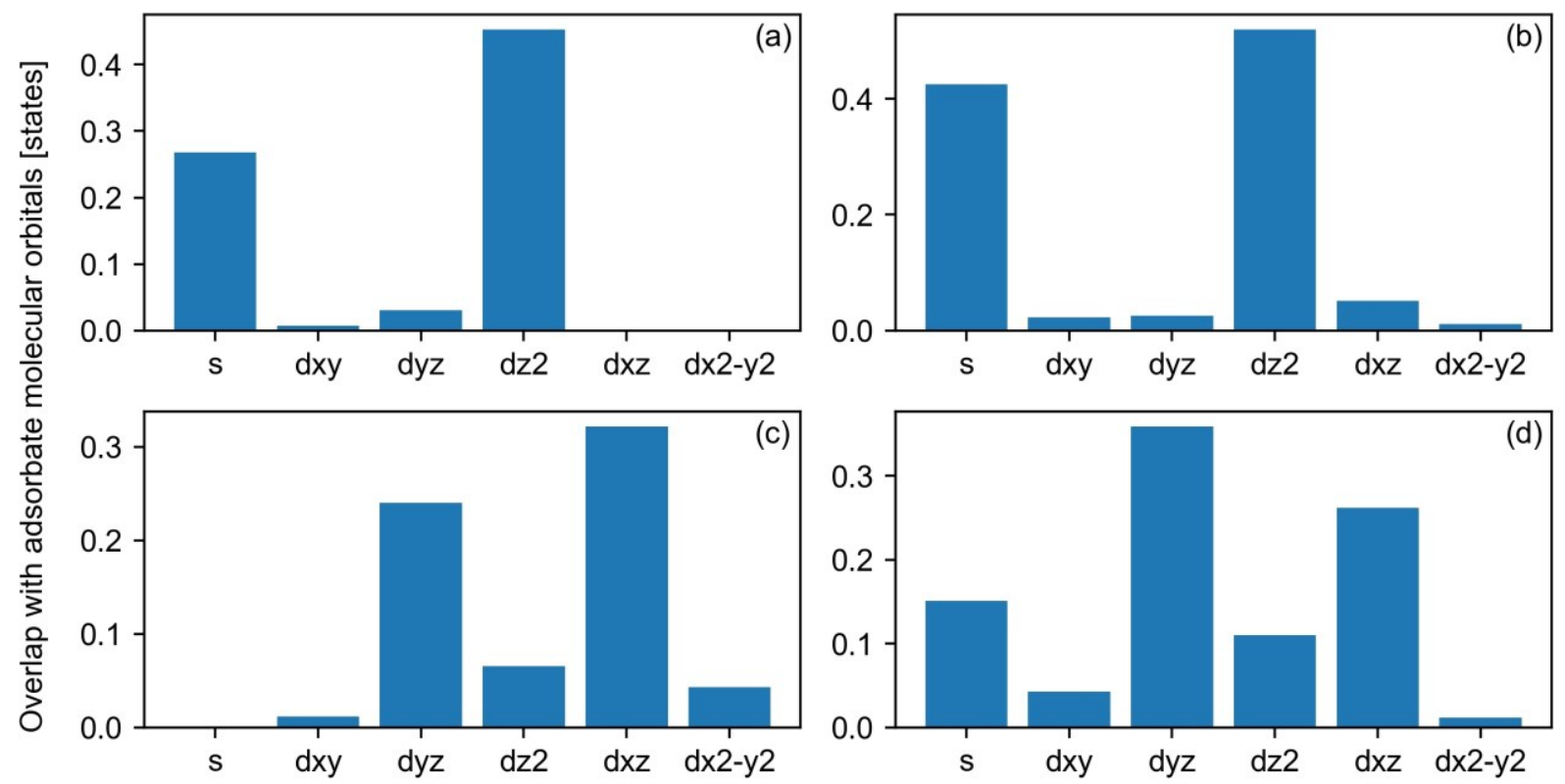

Metal atomic orbitals

Figure S8 Energy overlap of adsorbed NO molecular orbitals with Pt atomic orbitals at an atop site. Shown is the energy overlap of the metal atomic orbitals (Figure 4c) with the 2 (a), 3 (b), 4 (c), and 5 (d) molecular orbitals identified in Figure $4 \mathrm{~b}$ of adsorbed NO. Combined with the overlap populations and their integrals in Figure 5, interaction with molecular orbitals 3 or 4 will most likely dictate the $\mathrm{N}-\mathrm{O}$ frequencies across atop sites. 

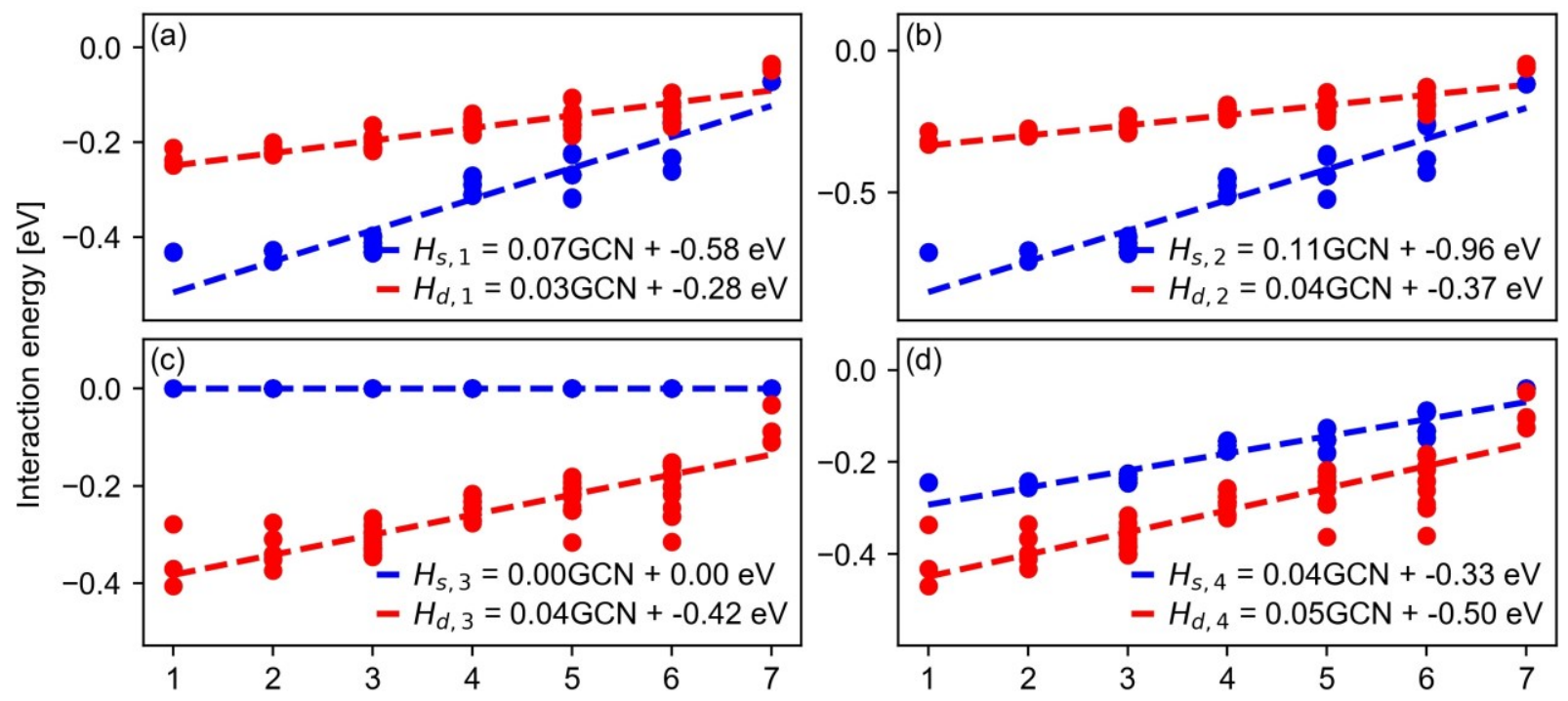

Generalized coordination number (GCN)

Figure S9 Interaction energy $(\mathrm{H})$ for $\mathrm{NO}$ at atop sites of various nanoparticles. Shown are the interaction energies of the metal atomic orbitals (Figure 4c) with the 2 (a), 3 (b), 4 (c), and 5 (d) molecular orbitals of adsorbed NO (Figure 4b). Also shown are the regressions of the $s$ (blue dashed lines) and $d$ (red dashed lines) interaction energies. Combined with the overlap populations and their integrals in Figure 4, it is clear that interaction with orbitals 3 and 5 will drive changes in $\mathrm{N}-\mathrm{O}$ frequencies at atop sites.

\section{Supplementary Note 5: NO on Pt(111) at a fcc 3-fold site}

Projected density of states and overlap population
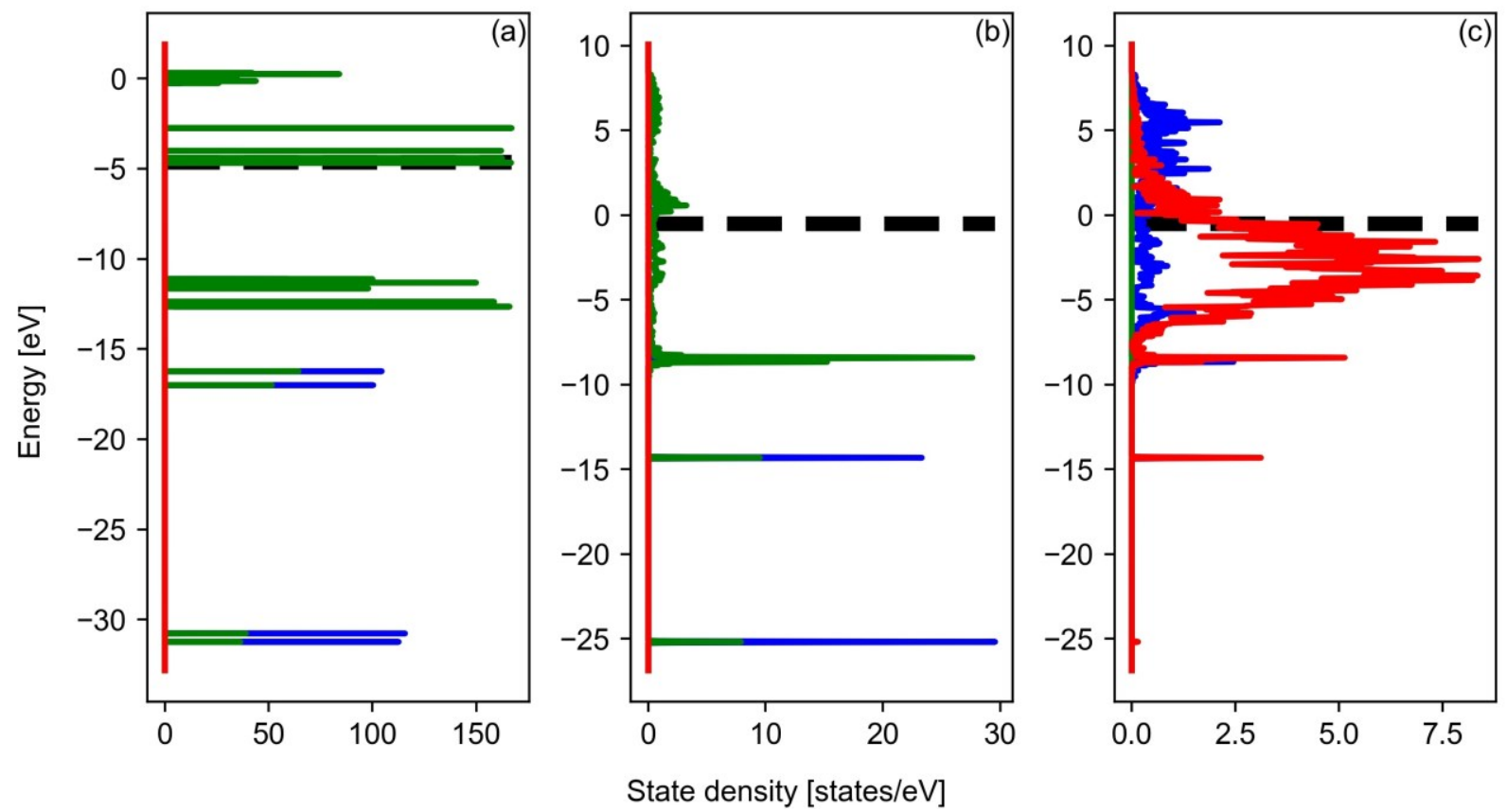

Figure S10 Projected density of states for NO at an fcc 3-fold site of Pt(111). Projected density of states for gas NO (a) compared to the projected density of $\mathrm{NO}$ at a fcc site (b) and that of the adsorption site after adsorption (c). Molecular orbitals of adsorbed $\mathrm{NO}$ in (b) have orbital energy centers located at $-25.19 \mathrm{eV},-14.32 \mathrm{eV},-8.70 \mathrm{eV},-8.50 \mathrm{eV},-8.42 \mathrm{eV}$, and $0.21 \mathrm{eV}$ with integrated state densities of $2.00,1.75,1.41,0.92,1.97$, and 7.96 , respectively. Density projected onto $\mathrm{s}, \mathrm{p}$, and $\mathrm{d}$ atomic orbitals are colored blue, green, and red, respectively. The fermi energy is given by the black dashed line. 

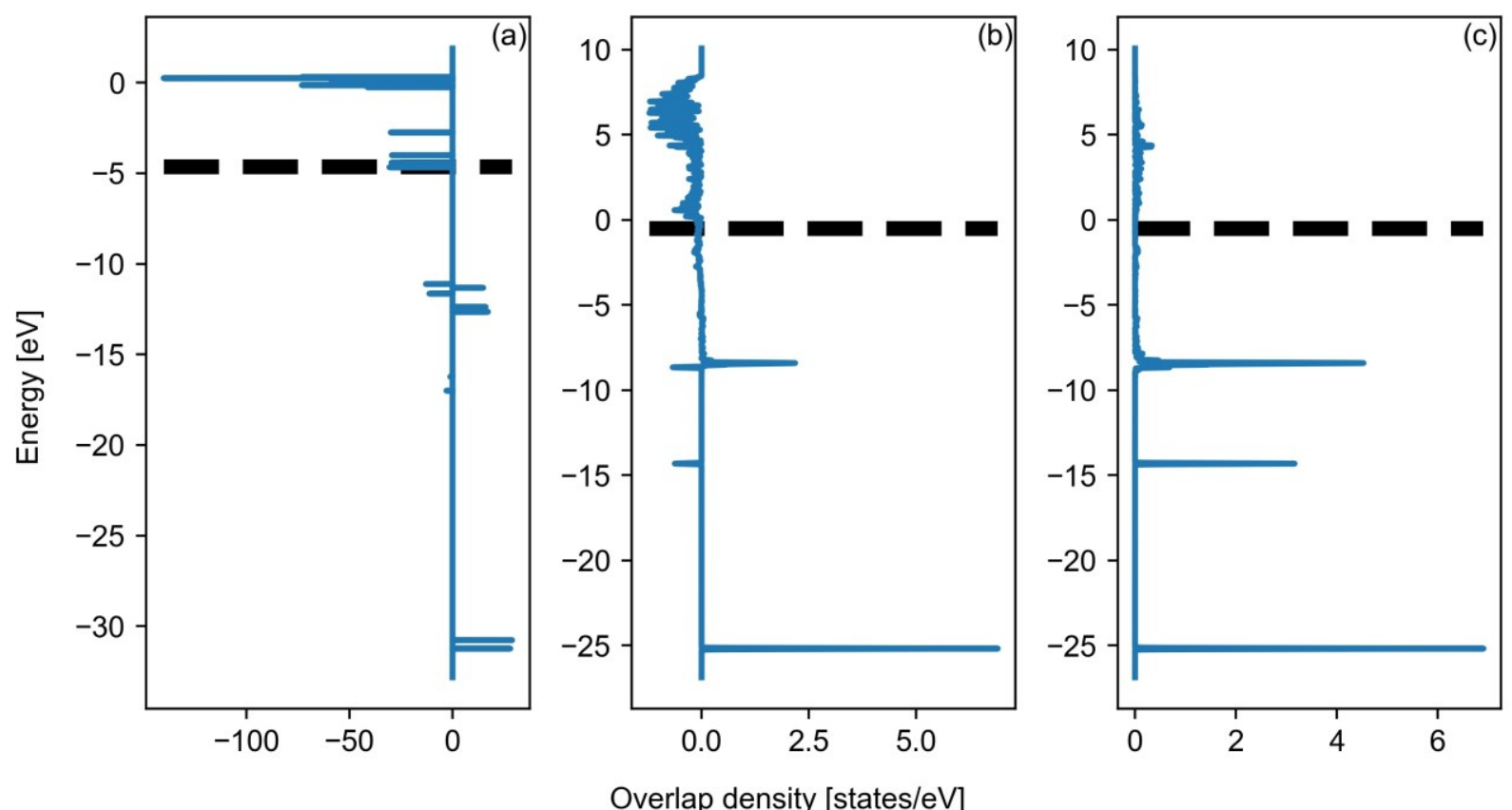

Figure S11 Crystal orbital overlap population analysis for NO on Pt(111) at an fcc 3-fold site. Shown are the overlap populations for gas NO (a), NO adsorbed at the fcc site (b), and the sum of the positive overlaps for the adsorbed NO and its overlap with the Pt adsorption site (c). The integrated overlap populations for molecular orbitals identified in Figure $8 \mathrm{~b}(1,2,3,4,5$, and 6$)$ are $0.37,-0.03,-0.04,0.05,0.15$, and -0.17 states up to the fermi level for adsorbed $N O$ (b), and $0.37,0.17,0.05,0.12,0.31$, and 0.12 states for the positive overlap of the combined NO and adsorption site, respectively. The overlap is given by the blue line and the fermi energy is given by the black dashed line.

Energy overlap integral and orbital interaction
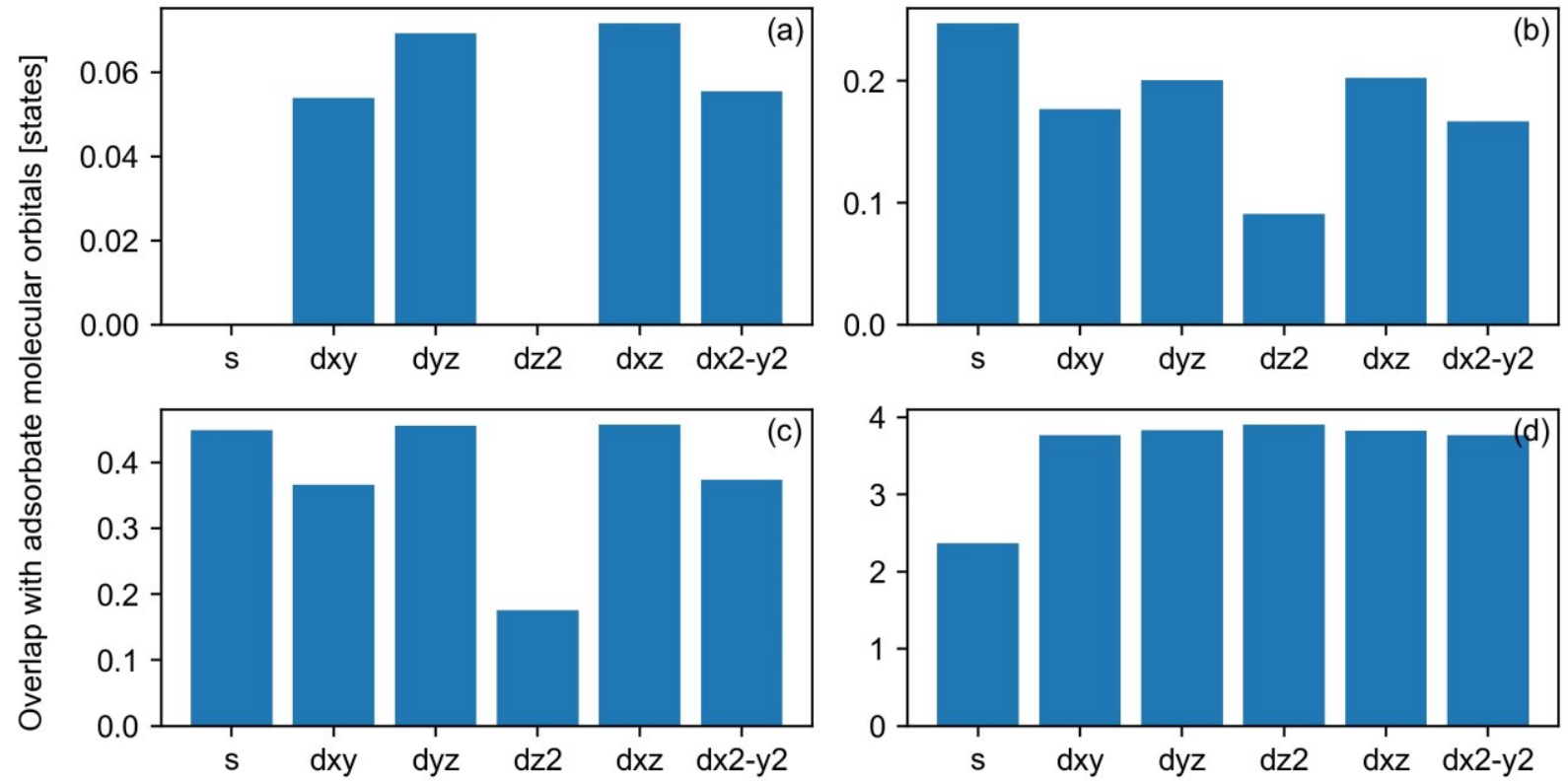

Metal atomic orbitals

Figure S12 Energy overlap of adsorbed NO molecular orbitals with Pt atomic orbitals at a fcc 3-fold site. Shown is the energy overlap of the metal atomic orbitals (Figure 8c) with the 1 (a), 4 (b), 5 (c), and 6 (d) molecular orbitals identified in Figure 8b of adsorbed NO. Combined with the overlap populations and their integrals in Figure 9, interaction with molecular orbital 5 will most likely dictate the $\mathrm{N}-\mathrm{O}$ frequencies across 3 -fold sites. 

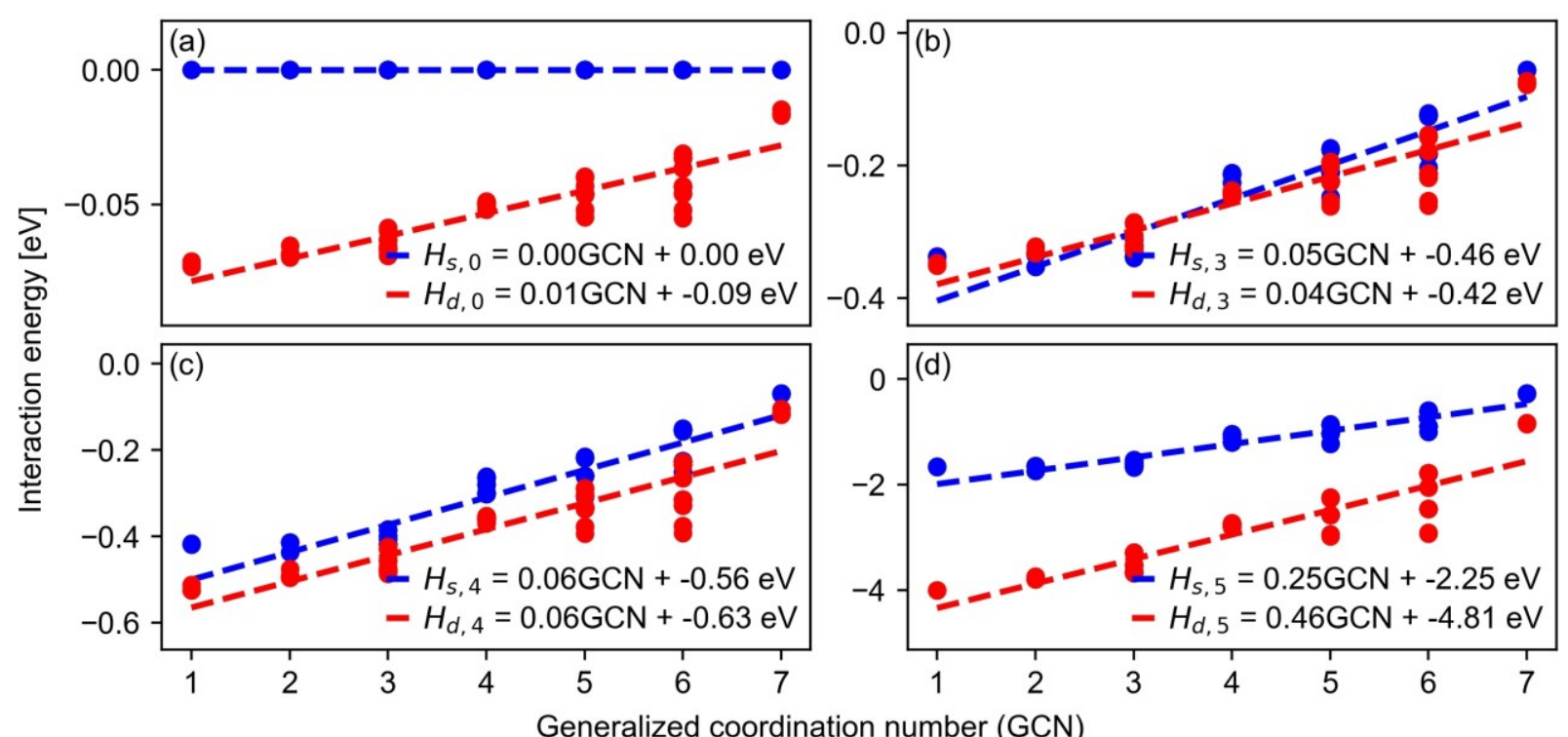

Figure S13 Interaction energy $(\mathrm{H})$ for $\mathrm{NO}$ at fcc 3-fold sites of various nanoparticles. Shown are the interaction energies of the metal atomic orbitals (Figure 8c) with the 1 (a), 4 (b), 5 (c), and 6 (d) molecular orbitals of adsorbed NO (Figure 8b). Also shown are the regressions of the $s$ (blue dashed lines) and $d$ (red dashed lines) interaction energies. Combined with the overlap populations and their integrals in Figure 9, interaction with both orbitals 5 and 6 will drive changes in $\mathrm{N}-\mathrm{O}$ frequencies at 3-fold sites.

\section{Supplementary Note 6: $\mathrm{C}_{\mathbf{2}} \mathrm{H}_{\mathbf{4}}$ on $\mathrm{Pt}(\mathbf{1 1 1})$ at an atop site}

Projected density of states and molecular orbitals and overlap population
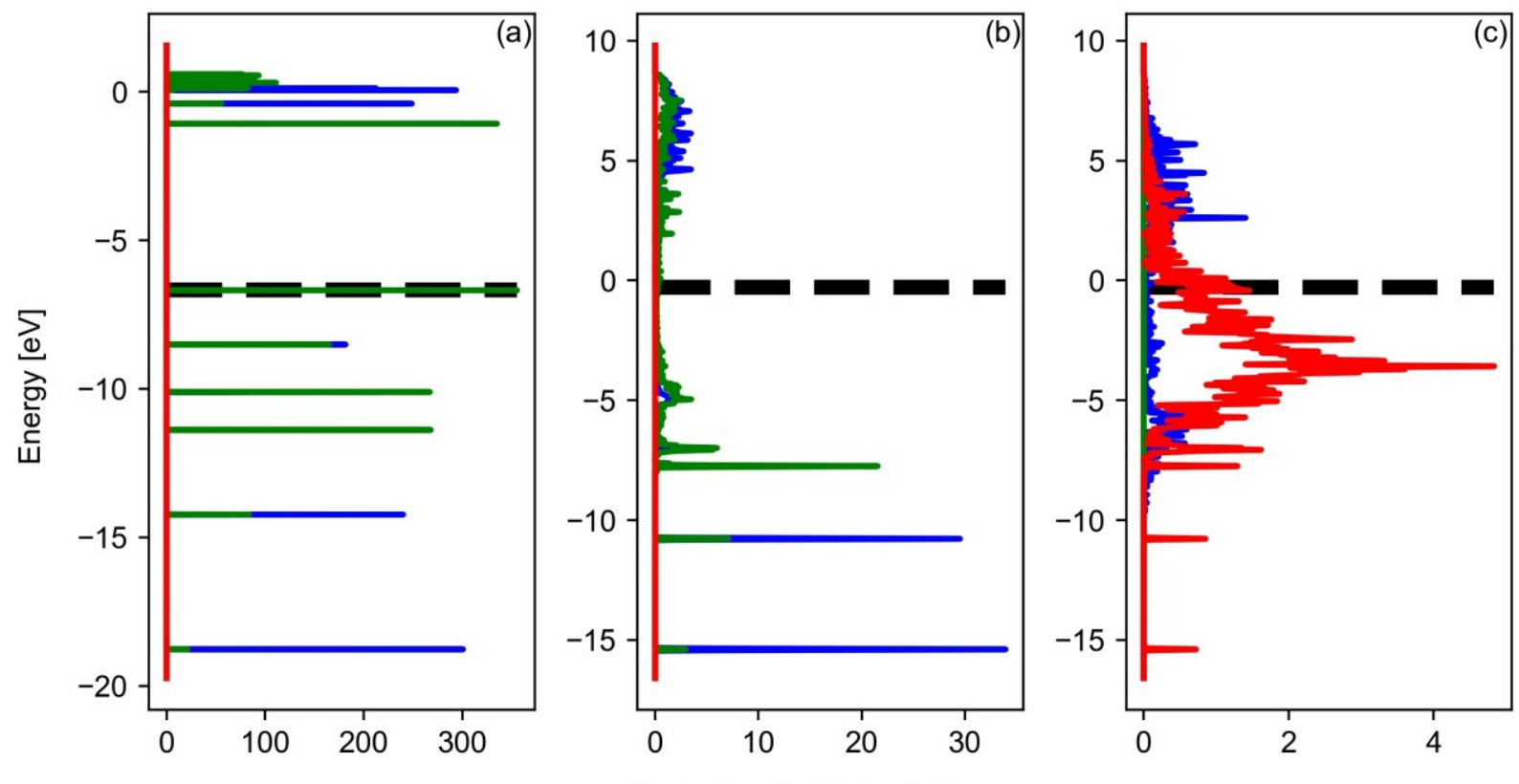

State density [states/eV]

Figure S14 Projected density of states for $\mathrm{C}_{2} \mathrm{H}_{4}$ at an atop site of Pt(111). Projected density of states for gas $\mathrm{C}_{2} \mathrm{H}_{4}(\mathrm{a})$ compared to the projected density of $\mathrm{C}_{2} \mathrm{H}_{4}$ at the atop site (b) and that of the adsorption site after adsorption (c). Molecular orbitals of adsorbed $\mathrm{C}_{2} \mathrm{H}_{4}$ with density below the fermi level in (b) have orbital energy centers located at $-15.39 \mathrm{eV},-10.78 \mathrm{eV},-7.73 \mathrm{eV},-$ $7.08 \mathrm{eV}, 2.41 \mathrm{eV}$, with integrated state densities of 1.96, 1.95, 1.95, 0.59, and 17.55, respectively. Density projected onto s, p, and $\mathrm{d}$ orbitals is blue, green, and red, respectively. The fermi energy is given by the black dashed line. 

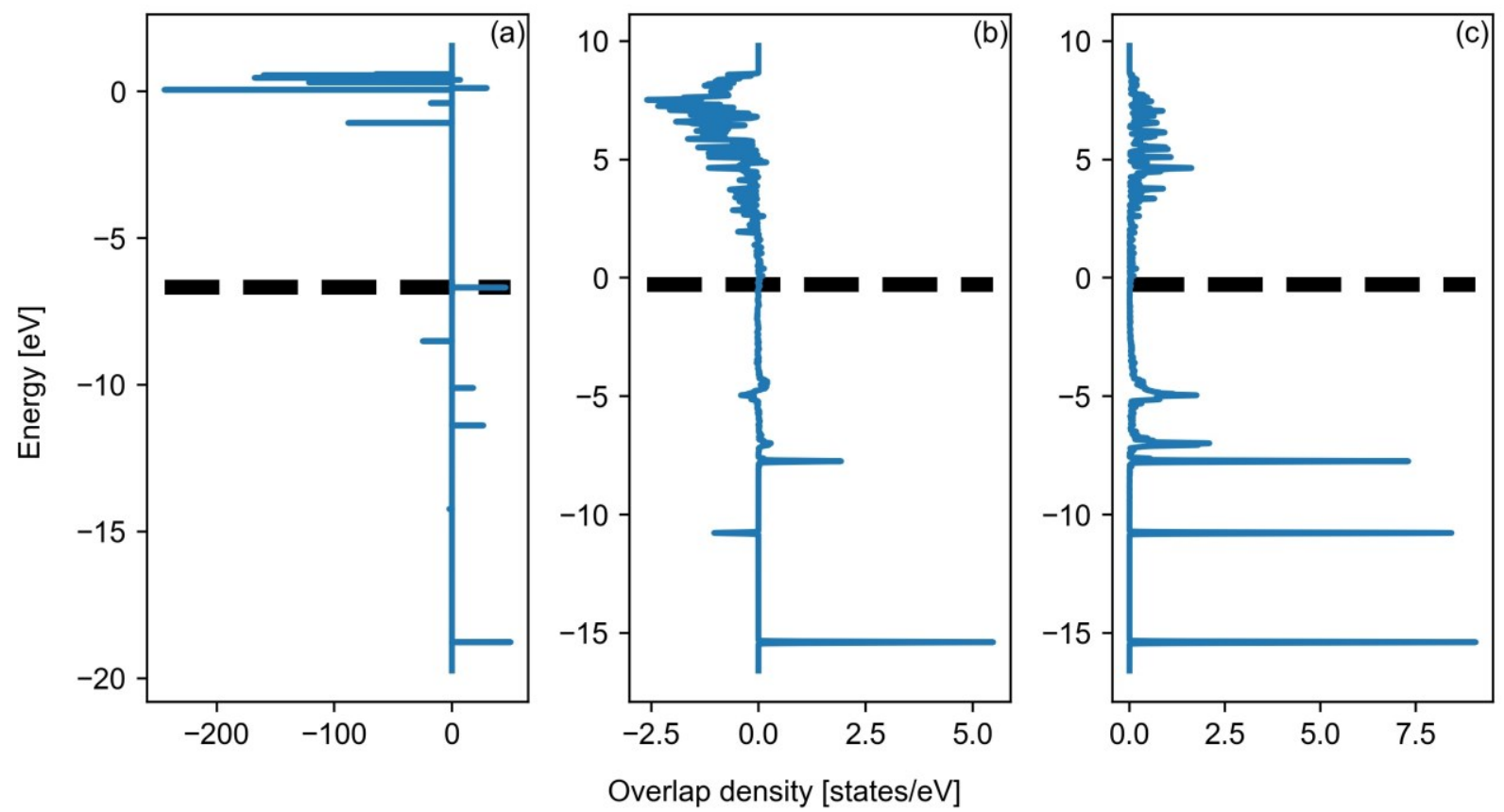

Figure $\mathrm{S} 15$ Crystal orbital overlap population analysis for $\mathrm{C}_{2} \mathrm{H}_{4}$ on $\mathrm{Pt}(111)$ at an atop site. Shown are the C-C overlap populations for gas $\mathrm{C}_{2} \mathrm{H}_{4}(\mathrm{a})$, the $\mathrm{C}$ - $\mathrm{C}$ overlap of $\mathrm{C}_{2} \mathrm{H}_{4}$ adsorbed at the atop site (b) and the sum of the positive overlaps for the adsorbed $\mathrm{C}_{2} \mathrm{H}_{4}$ and its overlap with the Pt adsorption site (c). The integrated C-C overlap populations for molecular orbitals identified in Figure $4 \mathrm{~b}(1,2,3,4$, and 5$)$ are $0.29,-0.05,0.13,0.01$, and 0.01 states up to the fermi level for adsorbed $\mathrm{C}_{2} \mathrm{H}_{4}(\mathrm{~b})$, and $0.48,0.45,0.49$, 0.13 , and 1.02 states for the positive overlap of the combined $\mathrm{C}_{2} \mathrm{H}_{4}$ and adsorption site, respectively. The overlap is given by the blue line and the fermi energy is given by the black dashed line.

Energy overlap integral and orbital interaction
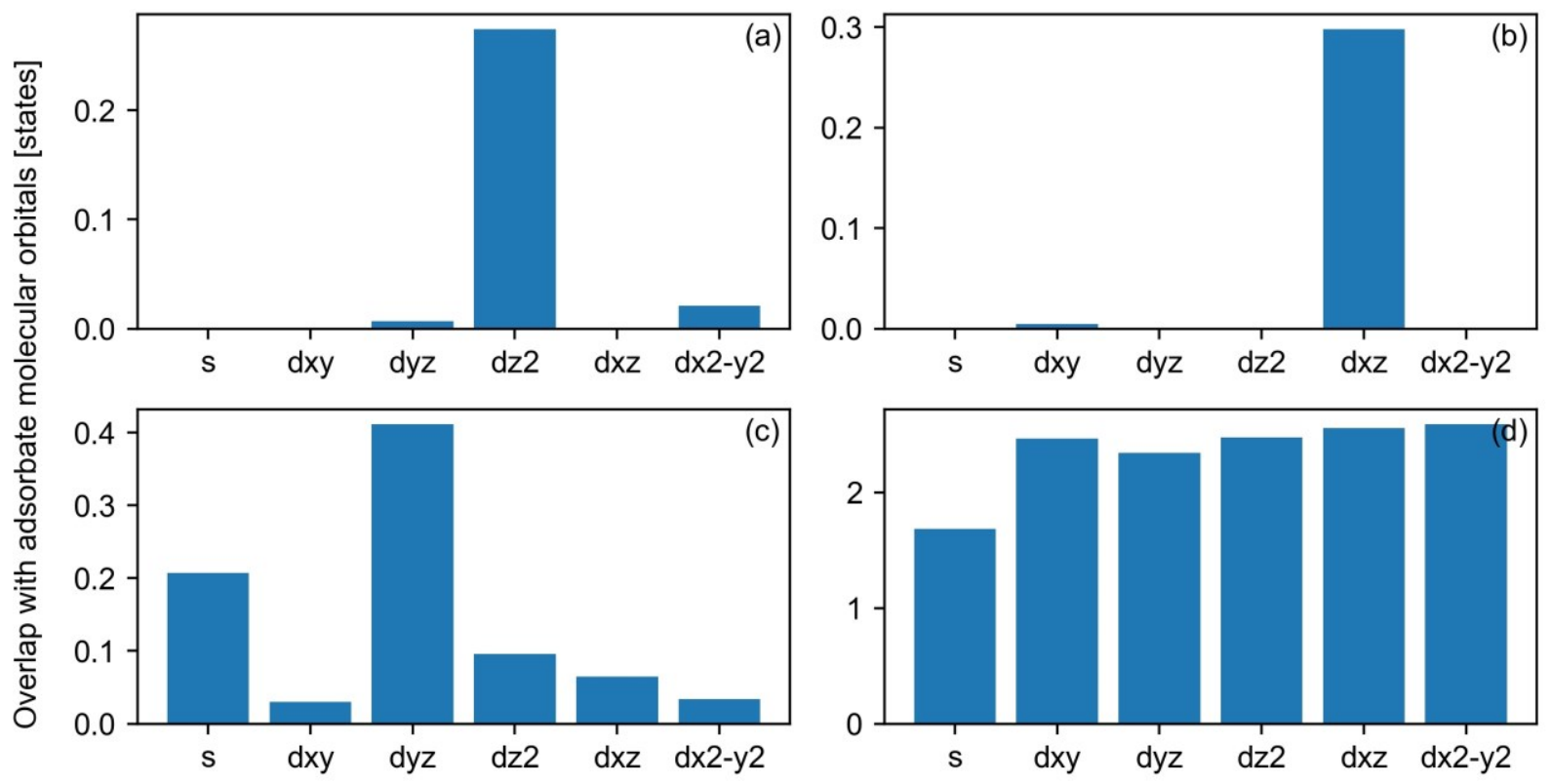

Metal atomic orbitals

Figure S16 Energy overlap of adsorbed $\mathrm{C}_{2} \mathrm{H}_{4}$ molecular orbitals with $\mathrm{Pt}$ atomic orbitals at an atop site. Shown is the energy overlap of the metal atomic orbitals (Figure 12c) with the 1 (a), 2 (b), 3 (c), and 5 (d) molecular orbitals identified in Figure $12 \mathrm{~b}$ of adsorbed $\mathrm{C}_{2} \mathrm{H}_{4}$. Combined with the overlap populations and their integrals in Figure 13, interaction with molecular orbitals 1,3 and 5 will all influence the $\mathrm{C}-\mathrm{C}$ frequencies across atop sites. 

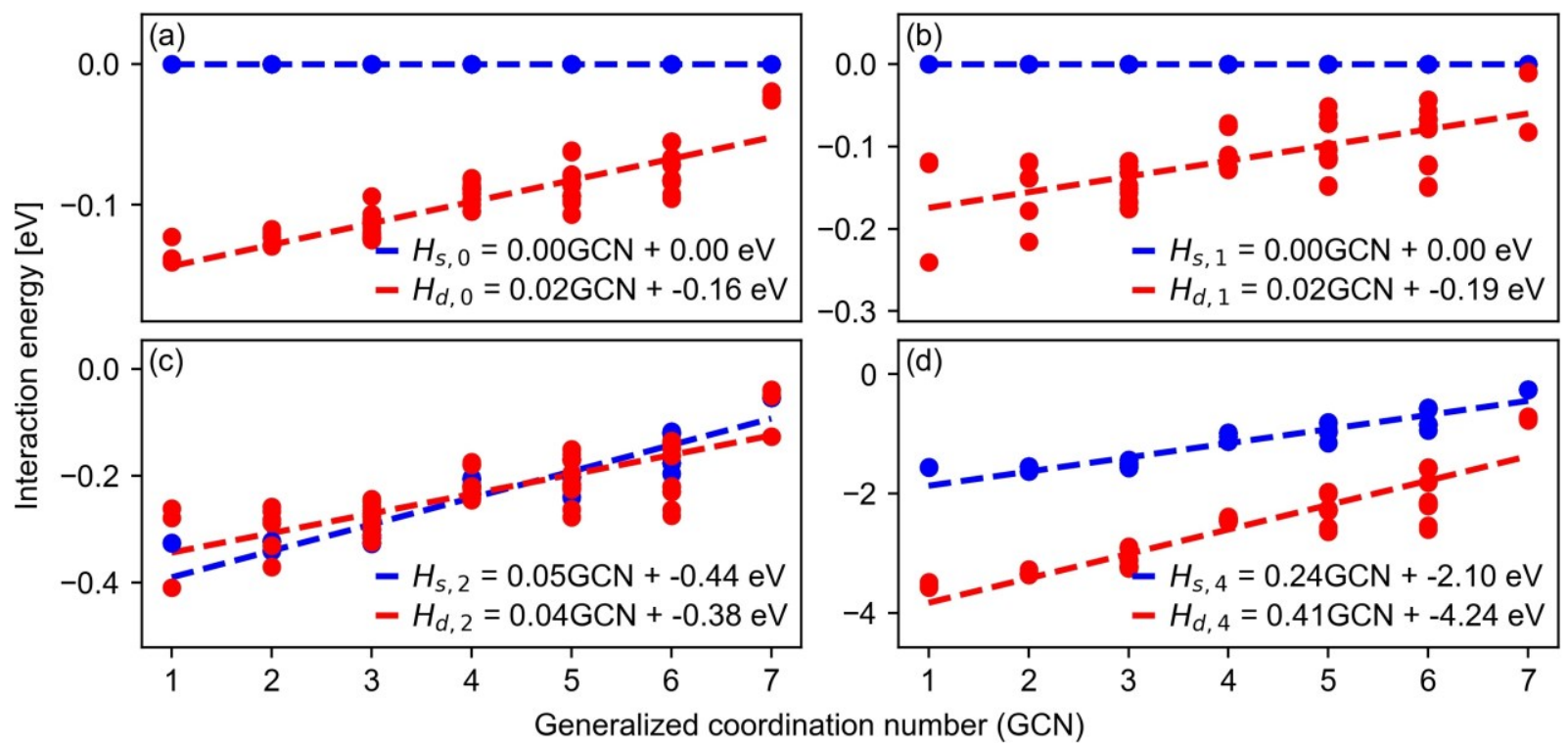

Figure $\mathrm{S} 17$ Interaction energy $(\mathrm{H})$ for $\mathrm{C}_{2} \mathrm{H}_{4}$ at atop sites of various nanoparticles. Shown are the interaction energies of the metal atomic orbitals (Figure 12c) with the 1 (a), 2 (b), 3 (c), and 5 (d) molecular orbitals of adsorbed $\mathrm{C}_{2} \mathrm{H}_{4}$ (Figure $12 \mathrm{~b}$ ). Also shown are the regressions of the $s$ (blue dashed lines) and $d$ (red dashed lines) interaction energies. Combined with the overlap populations and their integrals in Figure 13, interaction with both orbitals 3 and 5 will drive changes in C-C frequencies at atop sites.

\section{Supplementary Note 7: $\mathrm{C}_{2} \mathrm{H}_{4}$ on $\mathrm{Pt}(111)$ at a bridge site}

Projected density of states and molecular orbitals and overlap population
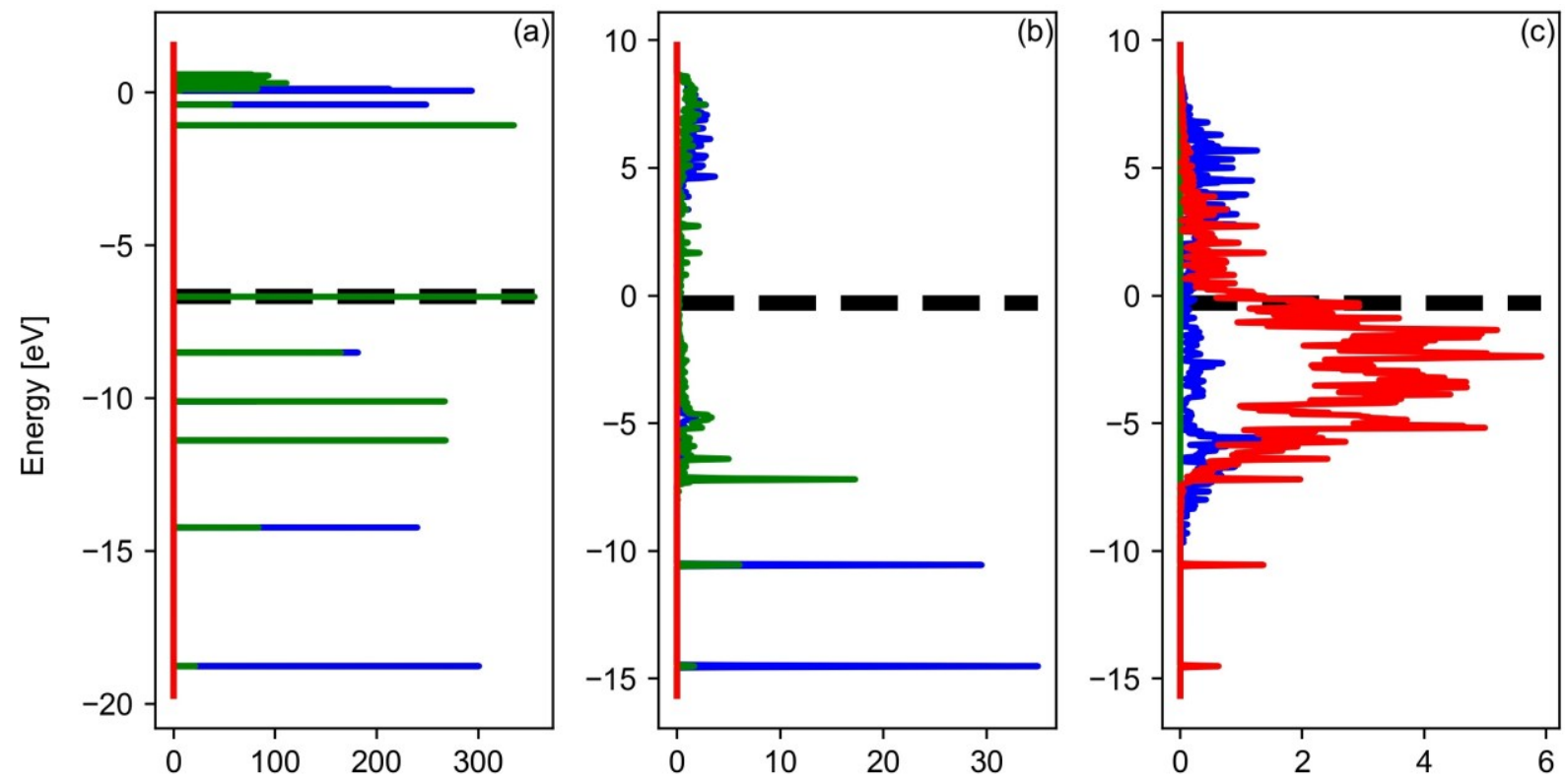

State density [states/eV]

Figure S18 Projected density of states for $\mathrm{C}_{2} \mathrm{H}_{4}$ at a bridge site of Pt(111). Projected density of states for gas $\mathrm{C}_{2} \mathrm{H}_{4}$ (a) compared to the projected density of $\mathrm{C}_{2} \mathrm{H}_{4}$ at the bridge site (b) and that of the adsorption site after adsorption (c). Orbitals of adsorbed $\mathrm{C}_{2} \mathrm{H}_{4}$ with density below the fermi level in (b) have orbital energy centers located at $-14.52 \mathrm{eV},-10.55 \mathrm{eV},-7.23 \mathrm{eV},-5.94 \mathrm{eV},-4.88 \mathrm{eV}$, and $1.57 \mathrm{eV}$ with integrated state densities of 1.95, 1.90, 1.69, 2.61, 0.55, and 9.67, respectively. Density projected onto $s, p$, and $\mathrm{d}$ atomic orbitals are colored blue, green, and red, respectively. 

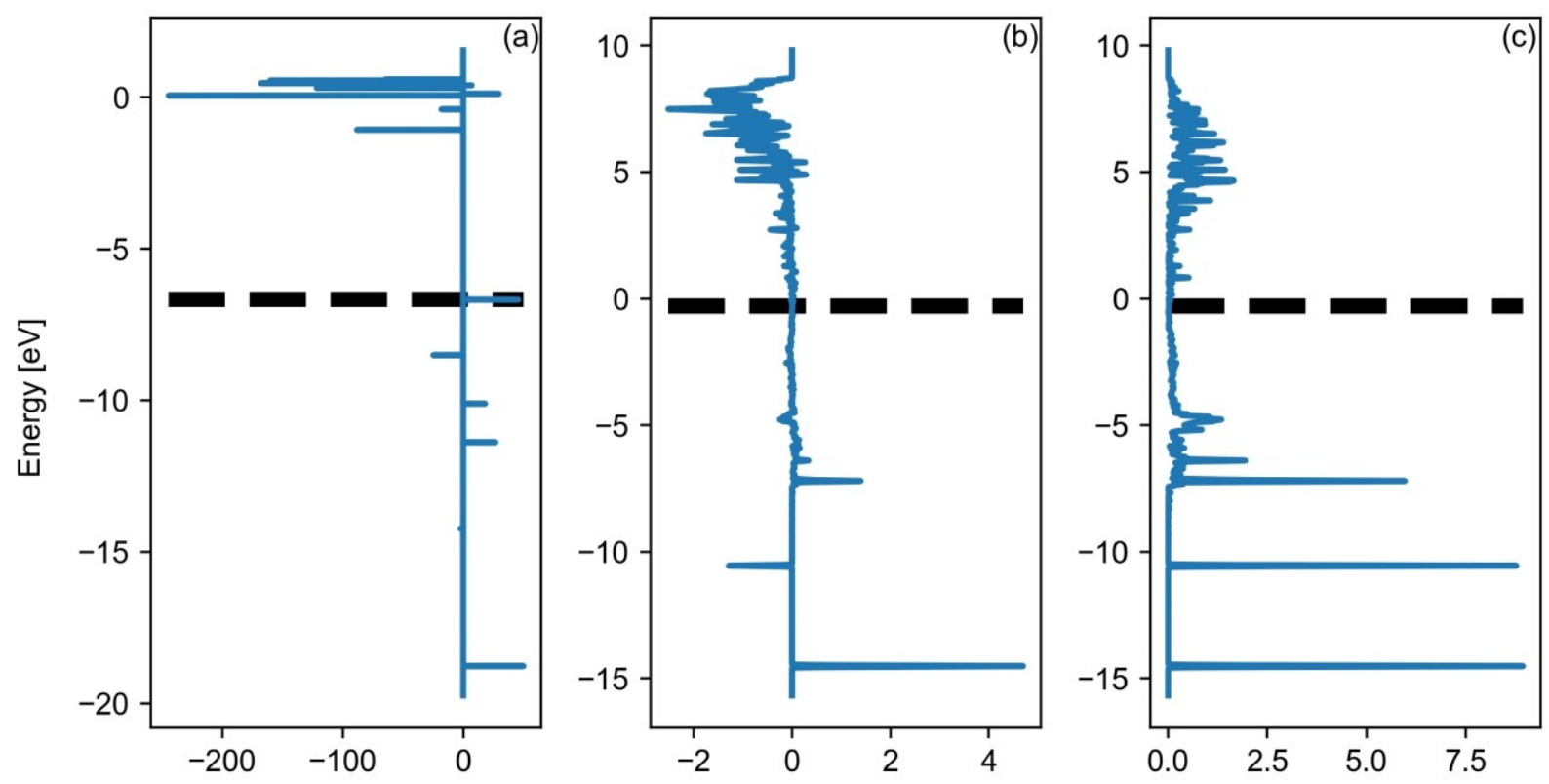

Overlap density [states/eV]

Figure S19 Crystal orbital overlap population analysis for $\mathrm{C}_{2} \mathrm{H}_{4}$ on $\mathrm{Pt}(111)$ at a bridge site. Shown are the C-C overlap populations for gas $\mathrm{C}_{2} \mathrm{H}_{4}(\mathrm{a})$, the $\mathrm{C}-\mathrm{C}$ overlap of $\mathrm{C}_{2} \mathrm{H}_{4}$ adsorbed at the bridge site (b) and the sum of the positive overlaps for the adsorbed $\mathrm{C}_{2} \mathrm{H}_{4}$ and its overlap with the Pt adsorption site (c). The integrated C-C overlap populations for molecular orbitals identified in Figure $4 \mathrm{~b}(1,2,3,4,5$, and 6$)$ are $0.25,-0.07,0.10,0.13,-0.01$, and -0.07 states up to the fermi level for adsorbed $\mathrm{C}_{2} \mathrm{H}_{4}(\mathrm{~b})$, and 0.48 , $0.47,0.43,0.66,0.12$, and 0.61 states for the positive overlap of the combined $\mathrm{C}_{2} \mathrm{H}_{4}$ and adsorption site, respectively. The overlap is given by the blue line and the fermi energy is given by the black dashed line.

Energy overlap integral and orbital interaction
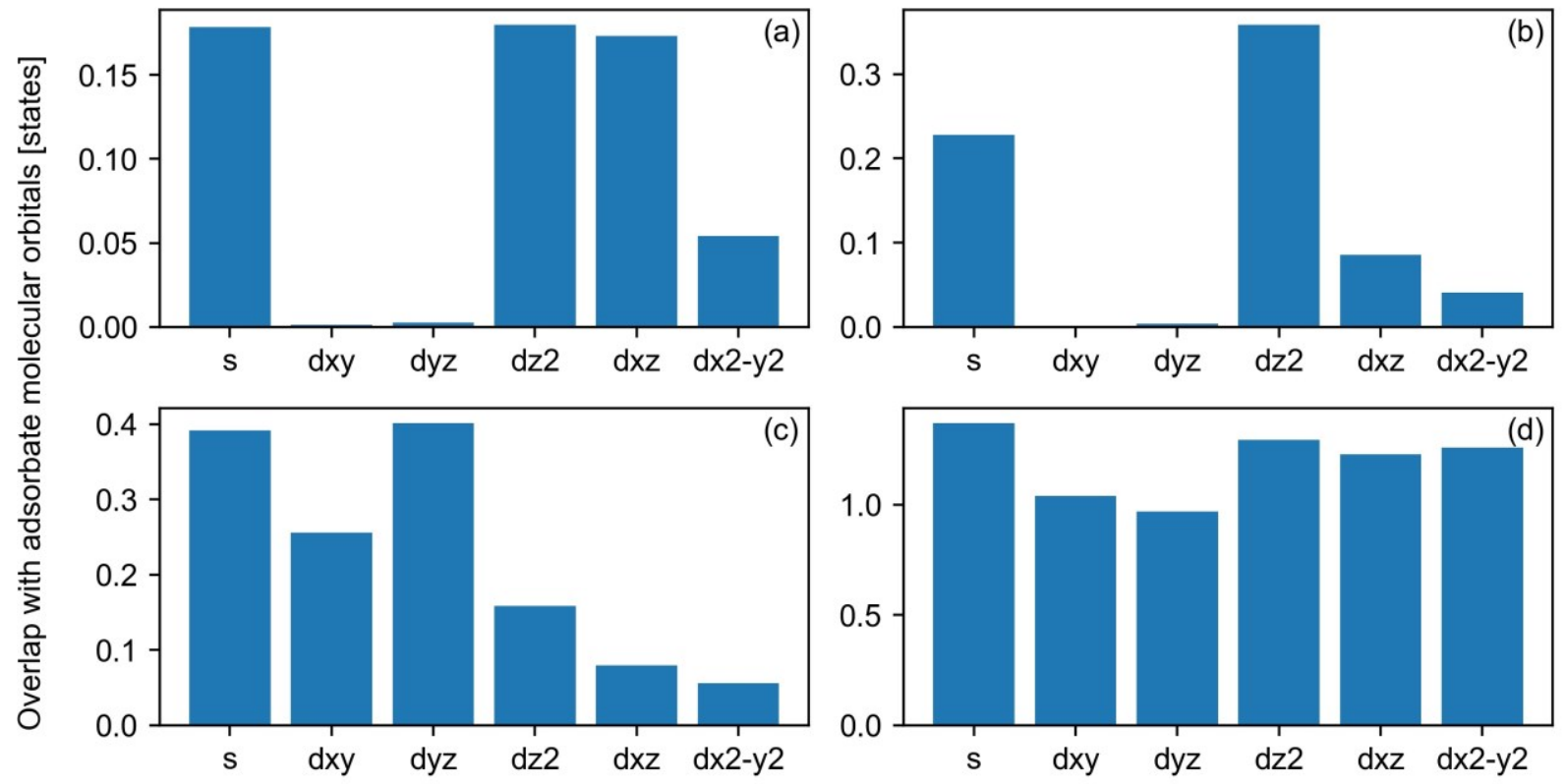

Metal atomic orbitals

Figure S20 Energy overlap of adsorbed $\mathrm{C}_{2} \mathrm{H}_{4}$ molecular orbitals with $\mathrm{Pt}$ atomic orbitals at a bridge site. Shown is the energy overlap of the metal atomic orbitals (Figure 16c) with the 1 (a), 2 (b), 3 (c), and 4 (d) molecular orbitals identified in Figure 16b of adsorbed $\mathrm{C}_{2} \mathrm{H}_{4}$. Combined with the overlap populations and their integrals in Figure 17, interaction with molecular orbitals 1,3 , and 4 will all influence the $\mathrm{C}-\mathrm{C}$ frequencies across bridge sites. 

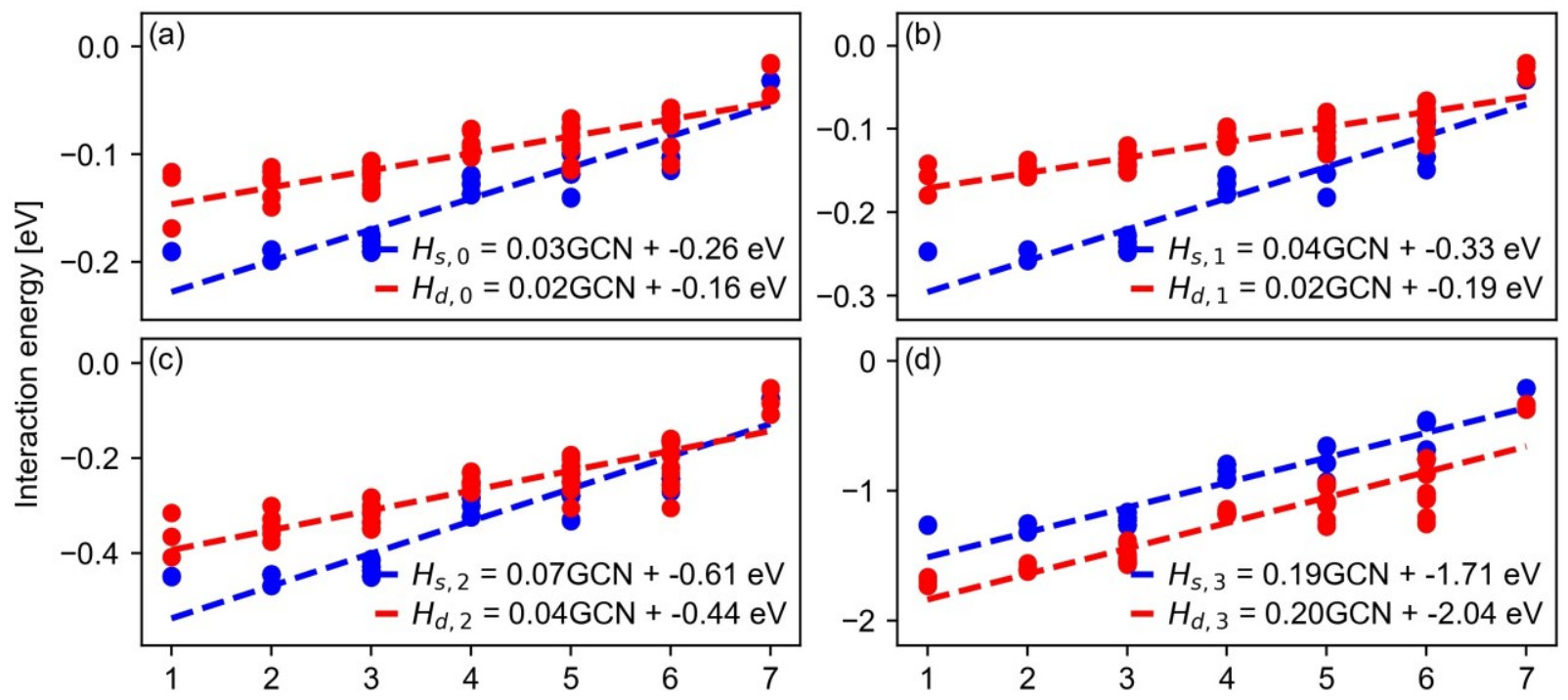

Generalized coordination number (GCN)

Figure $\mathrm{S} 21$ Interaction energy $(\mathrm{H})$ for $\mathrm{C}_{2} \mathrm{H}_{4}$ at bridge sites of various nanoparticles. Shown are the interaction energies of the metal atomic orbitals (Figure 16c) with the 1 (a), 2 (b), 3 (c), and 4 (d) molecular orbitals of adsorbed $\mathrm{C}_{2} \mathrm{H}_{4}$ (Figure 16b). Also shown are the regressions of the $s$ (blue dashed lines) and $d$ (red dashed lines) interaction energies. Combined with the overlap populations and their integrals in Figure 17, interaction with orbital 4 will drive changes in C-C frequencies across bridge sites.

\section{Supplementary Note 8: Gas energy shift}

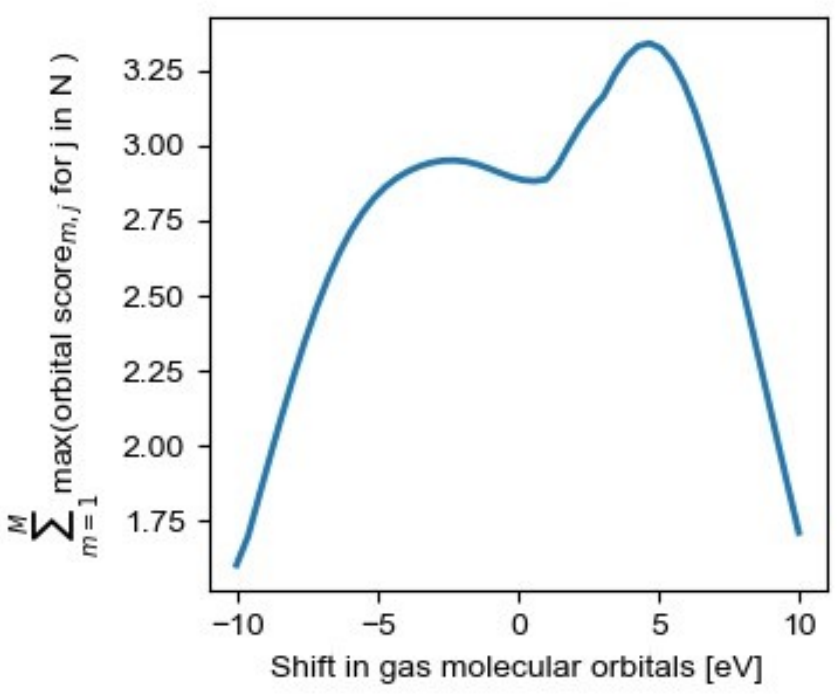

Figure S22 Sum of maximum orbital match scores for CO adsorbed on $\mathrm{Pt}(111)$ at an atop site as a function of the shift in the gas density of states energies. The most likely match occurs at a $4.64 \mathrm{eV}$ shift. 

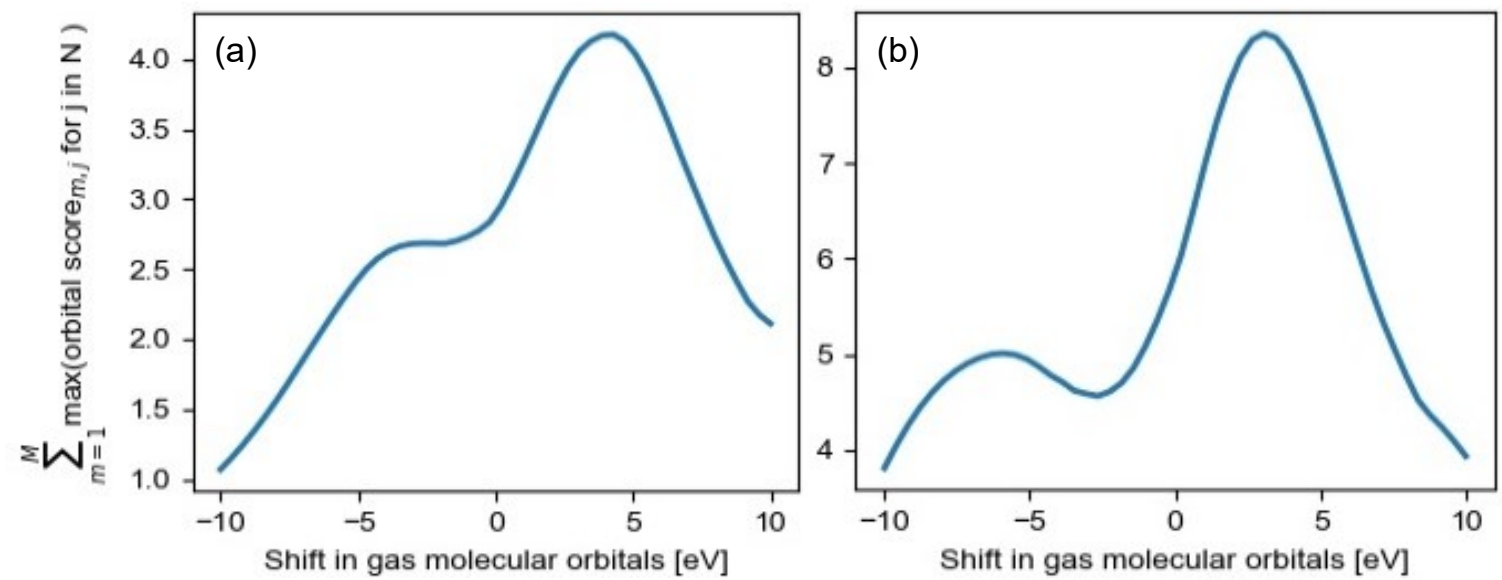

Figure S23 Sum of maximum orbital match scores for NO on $\mathrm{Pt}(111)$ as a function of the shift in the gas density of states energies. The most likely matches occurs at a 3.24 (a) and 3.26 (b) eV shift for NO at atop and fcc 3-fold sites, respectively.
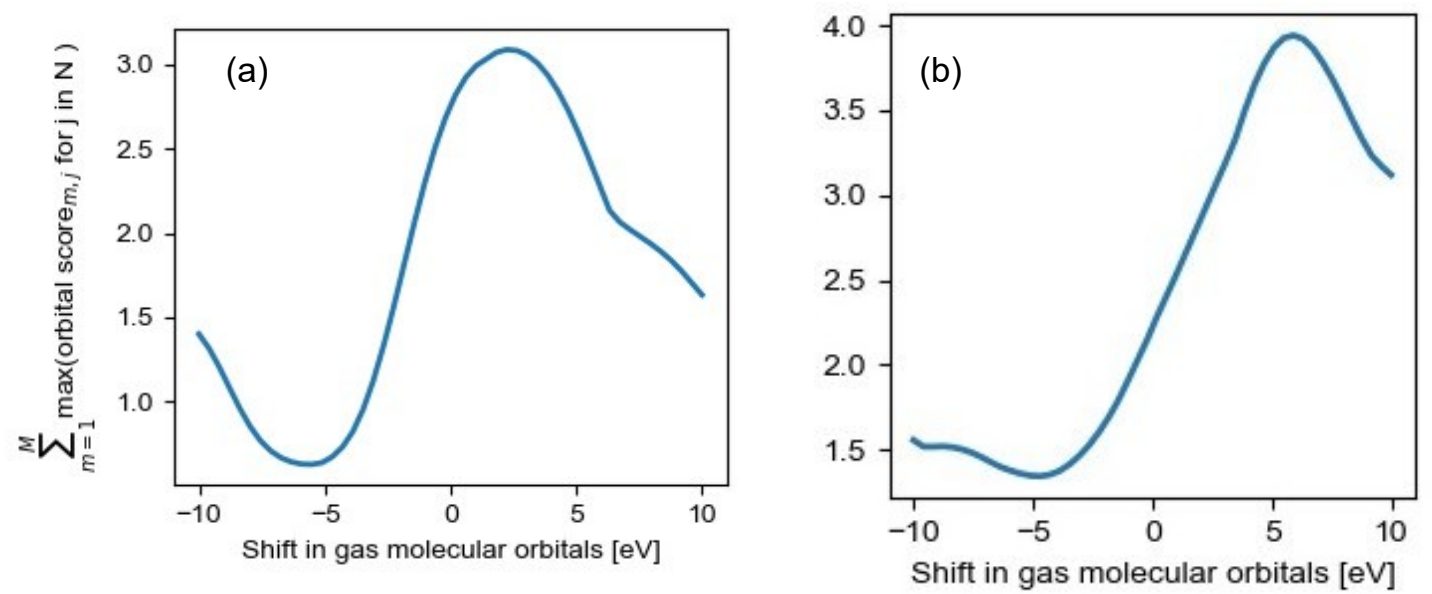

Figure S24 Sum of maximum orbital match scores for $\mathrm{C}_{2} \mathrm{H}_{4}$ on $\mathrm{Pt}(111)$ as a function of the shift in the gas density of states energies. The most likely matches occurs at a 2.34 (a) and 5.88 (b) eV shift for $\mathrm{C}_{2} \mathrm{H}_{4}$ at atop and bridges sites, respectively.

\section{Supplementary Note 9: Overview of $\mathrm{C}_{2} \mathrm{H}_{4}$ DFT data and structure model}

In Supplementary Figure S25, we show the distribution of the DFT data among different GCN groups. The meaning of the groups, and the method of partitioning data, is identical to that used in previous work ${ }^{8}$. 


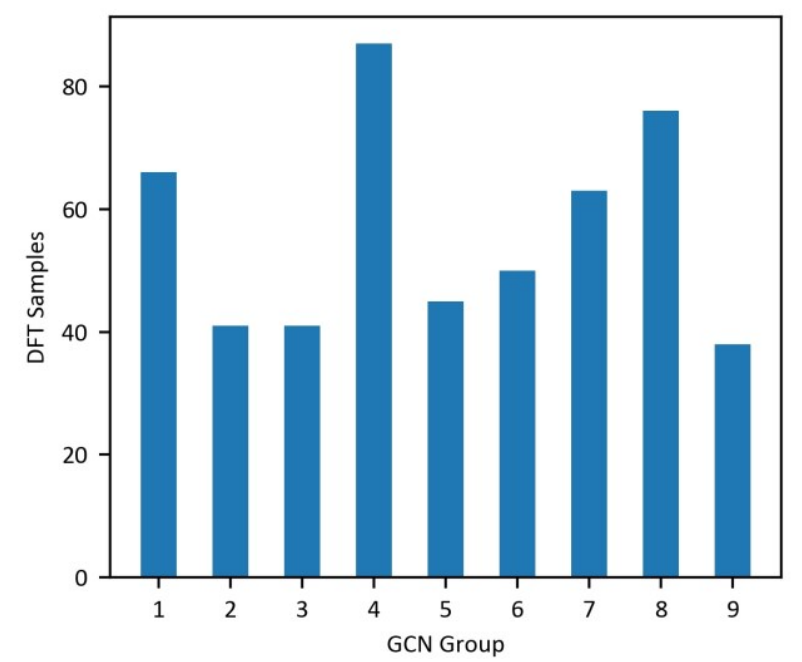

Figure S25 Binning of GCN values from primary zero-coverage data into GCN groups via k-means clustering. The [GCN group, GCN range] are as follows: [1, 0-1.8], [2, 1.8-2.9], [3, 2.9-3.7], [4, 3.7-4.3], [5, 4.3-4.9], [6, 4.9-5.4], [7, 5.5-6.1], [8, 6.2-7.1], [9, 7.18.5].

Two hundred randomly picked samples of binding-type model predictions on a holdout test set are given in Supplementary Figure S26. Shown are the actual (x-axis) and predicted ( $y$-axis) occupation of atop (blue circles) and bridge (orange squares) binding-types as a percentage of the total occupied sites by $\mathrm{C}_{2} \mathrm{H}_{4}$. The error is uniformly low.

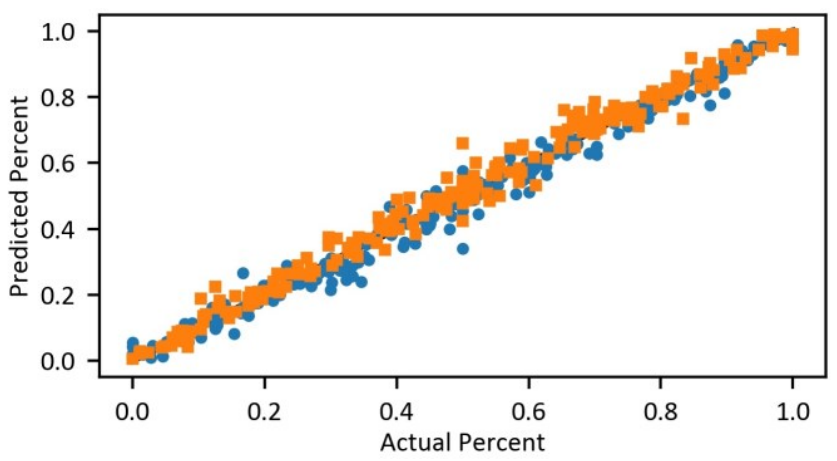

Figure S26 Assessment of low coverage $\mathrm{C}_{2} \mathrm{H}_{4}$ binding-type model. Parity plot of predicted vs. actual binding pdfs for synthetic spectra of $\mathrm{C}_{2} \mathrm{H}_{4}$ adsorption on $\mathrm{Pt}$ at high coverage. Atop (blue circles), bridge (orange squares) binding-types.

\section{Supplementary Note 10: Deriving $\chi_{\mu}(E)$ and $E_{\mu \nu}$ from COOP}

In order to replace $S_{\mu \nu}$ in Equation 2 of the main text with a value that is a function of energy $(E)$, we turn to the integrated COOP restricted to interaction between orbitals $\mu$ and $\nu$. The COOP is given by ${ }^{9}$

$$
\operatorname{COOP}_{\mu \nu}(E)=S_{\mu \nu} \sum_{j, \vec{k}} C_{\mu, j}^{*}(\vec{k}) C_{v, j}(\vec{k}) \delta\left(\varepsilon_{j}(\vec{k})-E\right),
$$

where $S_{\mu \nu}$ is the overlap integral, $\varepsilon_{j}$ is energy and band j, $\vec{k}$ is the k-point index, and $C^{\prime}$ s are the coefficients in the linear combination of atomic orbitals formalism ${ }^{10-11}$. The local density-of-states (LDOS) projected onto $\mu$ at site $(i)$ is then $^{9,12}$

$$
\operatorname{LDOS}_{\mu \mu}(E)=\rho_{i, \mu}(E)=\sum_{j, \overrightarrow{\vec{k}}} C_{\mu, j}^{*}(\vec{k}) C_{\mu, j}(\vec{k}) \delta\left(\varepsilon_{j}(\vec{k})-E\right)
$$


If the system is large or a single k-point is used, Equations 31 and 32 simplify to

$$
\operatorname{COOP}_{\mu \nu}(E)=S_{\mu \nu} C_{\mu, j}^{*}(E) C_{\nu, j}(E),
$$

and

$$
\rho_{i, \mu}(E)=C_{\mu, j}^{*}(E) C_{\mu, j}(E)
$$

As we are interested in only the bonding (i.e., positive) portion of the COOP, which is real valued, we substitute the simplified orbital projected LDOS into the COOP such that

$$
\operatorname{COOP}_{\mu \nu}(E)=S_{\mu \nu} \sqrt{\rho_{i, \mu}(E)} \sqrt{\rho_{a, v}(E)} .
$$

To replace $S_{\mu \nu}$ of Equation 2 in the main text with the energy overlap of $\mu$ with $v$, it must be replaced by

$$
\frac{\int_{-\infty}^{E_{F}} \operatorname{COOP}_{\mu \nu}(E)}{S_{\mu \nu}}=E_{\mu \nu}=\int_{-\infty}^{E_{F}}\left(\sqrt{\rho_{i, \mu}(E)} \sqrt{\rho_{a, v}(E)}\right) d E .
$$

Equation 5 of the main text then arises from treating $\sqrt{\rho_{i, \mu}(E)}$ as an orbital in energy space where $E_{F}$ is the fermi energy.

\section{Supplementary References}

1. Paxton, A. T.; Sutton, A. P.; Nex, C. M. M., Structural Stability of Silicon in Tight-Binding Models. J. Phys. C: Solid State Phys. 1987, 20, L263-L269.

2. Wu, D.; Xi, C.; Dong, C.; Liu, H.; Du, X.-W., Bond-Energy-Integrated Coordination Number: An Accurate Descriptor for Transition-Metal Catalysts. J. Phys. Chem. C 2019, 123, 28248-28254.

3. Hammer, B., Special Sites at Noble and Late Transition Metal Catalysts. Top. Catal. 2006, 37, 3-16.

4. Xin, H.; Vojvodic, A.; Voss, J.; Nørskov, J. K.; Abild-Pedersen, F., Effects of D-Band Shape on the Surface Reactivity of Transition-Metal Alloys. Phys. Rev. B 2014, 89, 115114.

5. Ma, X.; Xin, H., Orbitalwise Coordination Number for Predicting Adsorption Properties of Metal Nanocatalysts. Phys. Rev. Lett. 2017, 118, 036101.

6. Xi, C.; Zou, C.; Wang, M.; Wu, D.; Liu, H.; Dong, C.; Du, X.-W., A Bond-Energy-Integrated-Based Descriptor for High-Throughput Screening of Transition Metal Catalysts. J. Phys. Chem. C 2020, 124, 5241-5247.

7. Vojvodic, A.; Nørskov, J. K.; Abild-Pedersen, F., Electronic Structure Effects in Transition Metal Surface Chemistry. Top. Catal. 2014, 57, 25-32.

8. Lansford, J. L.; Vlachos, D. G., Infrared Spectroscopy Data- and Physics-Driven Machine Learning for Characterizing Surface Microstructure of Complex Materials. Nat. Commun. 2020, 11, 1513.

9. Maintz, S.; Deringer, V. L.; Tchougréeff, A. L.; Dronskowski, R., Analytic Projection from Plane-Wave and Paw Wavefunctions and Application to Chemical-Bonding Analysis in Solids. J. Comput. Chem. 2013, 34, 2557-2567.

10. Chen, M.; Waghmare, U. V.; Friend, C. M.; Kaxiras, E., A Density Functional Study of Clean and HydrogenCovered A-Moo3(010): Electronic Structure and Surface Relaxation. J. Chem. Phys. 1998, 109, 6854-6860.

11. Szabo, A.; Ostlund, N. S., Modern Quantum Chemistry: Introduction to Advanced Electronic Structure Theory. Courier Corporation: 2012.

12. Steinberg, S.; Dronskowski, R., The Crystal Orbital Hamilton Population (Cohp) Method as a Tool to Visualize and Analyze Chemical Bonding in Intermetallic Compounds. Crystals 2018, 8, 225. 